\title{
Numerical investigation of structural behavior during fluid excited vibrations
}

\author{
Saim Yigit - Michael Schäfer - Marcus Heck \\ Department of Numerical Methods in Mechanical Engineering \\ Technische Universität Darmstadt, Petersenstr. 30, 64287 Darmstadt, Germany \\ \{yigit, schaefer, heck\}@fnb.tu-darmstadt.de
}

\begin{abstract}
In the present paper different occurring phenomena during the interaction between certain structural configurations and laminar incompressible flows are investigated. Preliminary investigations concerning the grid movement technique provide the basis for the adequate treatment of the fluid structure interaction problems. Several mechanisms according to real experiments are presented. Systematical numerical studies of material parameters are performed on the basis of a moderately complex fluid structure interaction test configuration. The solution procedure involves the finite-volume flow solver FASTEST, the finite-element structural solver FEAP, and the coupling interface MpCCI.

RÉSUMÉ. Dans cet article des phénomènes différents apparaissant pendant l'interaction entre certaines configurations structurales et des écoulements laminaires et incompressibles sont explorés. Des investigations précédentes concernant la technique de mouvement du maillage fournissent la base pour le traitement adéquat des problèmes d'interaction fluide structure. Conformément aux expériences réelles divers mécanismes sont représentés. A la base des configurations d'interaction fluide structure de test faiblement complexe des études numériques des paramètres matériaux sont effectuées systématiquement. La procédure de résolution inclut le solveur d'écoulement du fluide basé sur la méthode des volumes finis FASTEST, le solveur structural basé sur la méthode des éléments finis FEAP et l'interface de couplage MpCCI.

KEYWORDS: FSI, large deformations, fluid excited vibrations, elliptic grid generation.

MOTS-CLÉS : interaction fluide structure, grandes déformations, vibrations excitées par le fluide, maillage elliptique.
\end{abstract}

DOI:10.3166/REMN.16.491-519@ 2007 Lavoisier, Paris. Tous droits réservés

REMN - 16/2007. Fluid structure interaction, pages 491 to 519 


\section{Introduction}

The interaction of fluids and deformable bodies often cause complex physical sequences occurring in many applications in industry and science. Besides the task of solving the coupled problem from the mathematical point of view, a further challenge exists in understanding and describing the corresponding mechanisms. Most of these problems are accompanied by large structural deformations leading to highly nonlinear phenomena where the classical theory of small oscillations becomes invalid.

In the present paper such kind of coupled problems involving laminar incompressible flows are investigated with respect to the mechanical behavior and the coupling mechanisms. The numerical solution of the coupled problem is done by an implicit partitioned solution approach. The method is realized on the basis of the finite-volume flow solver FASTEST (FASTEST, 2004), the finite-element structural solver FEAP (Taylor, 2002), and the coupling interface library MpCCI (SCAI, 2004). To stabilize the whole solution procedure an underrelaxation technique is employed determining the magnitude of the coupling scheme. In addition, multigrid techniques for accelerating the computations are applied for the fluid part. For the mesh movement algebraic and elliptic approaches are considered, which are investigated comparatively in a preliminary study.

\section{Governing equations}

We consider a problem domain $\Omega$ consisting of a fluid part $\Omega_{\mathrm{f}}$ and a solid part $\Omega_{\mathrm{s}}$, which regarding the shape as well as the location of fluid and solid parts can be arbitrary. For the fluid domain part $\Omega_{\mathrm{f}}$ we assume a flow of an incompressible Newtonian fluid. In this case the basic conservation equations governing transport of mass and momentum for a fluid control volume $V_{\mathrm{f}}$ with surface $S_{\mathrm{f}}$ are given by:

$$
\begin{aligned}
& \int_{S_{\mathrm{f}}}\left(v_{j}-v_{j}^{\mathrm{g}}\right) n_{j} \mathrm{~d} S_{\mathrm{f}}=0, \\
& \frac{\mathrm{d}}{\mathrm{d} t} \int_{V_{\mathrm{f}}} \rho_{\mathrm{f}} v_{i} \mathrm{~d} V_{\mathrm{f}}+\int_{S_{\mathrm{f}}}\left[\rho_{\mathrm{f}} v_{j}\left(v_{i}-v_{i}^{\mathrm{g}}\right) n_{j}-T_{i j} n_{j}\right] \mathrm{d} S_{\mathrm{f}}=\int_{V_{\mathrm{f}}} \rho_{\mathrm{f}} f_{\mathrm{f} i} \mathrm{~d} V_{\mathrm{f}},
\end{aligned}
$$

where $v_{i}$ is the velocity vector with respect to Cartesian coordinates $x_{i}, t$ is the time, $\rho_{\mathrm{f}}$ is the fluid density, and $f_{\mathrm{f} i}$ are external body forces (e.g., buoyancy forces). $v_{i}^{\mathrm{g}}$ is the velocity with which $S_{\mathrm{f}}$ may move (grid velocity) due to displacements of solid parts. The stress tensor $T_{i j}$ for incompressible Newtonian fluids with the pressure $p$ and the dynamic viscosity $\mu_{\mathrm{f}}$ is defined by:

$$
T_{i j}=\mu_{\mathrm{f}}\left(\frac{\partial v_{j}}{\partial x_{i}}+\frac{\partial v_{i}}{\partial x_{j}}\right)-p \delta_{i j}
$$


The model equations for the solid domain $\Omega_{\mathrm{s}}$ may take rather different forms depending on the concrete problem and coupling mechanisms involved. The models range from a simple rigid body motion without any deformation of the solid up to strongly nonlinear (physically and/or geometrically) deformations. The basic balance equation for momentum for the solid domain $\Omega_{\mathrm{s}}$ can be written as

$$
\rho_{\mathrm{s}} \ddot{u}_{i}-\frac{\partial \sigma_{i j}}{\partial x_{j}}=\rho_{\mathrm{s}} f_{\mathrm{s} i}
$$

where $u_{i}$ is the displacement, $\sigma_{i j}$ denotes the Cauchy stress tensor, $\rho_{\mathrm{s}}$ is the density of the solid, and $f_{\mathrm{s} i}$ are external volume forces acting on the solid (e.g., gravitational forces).

The solid model equations are completed by a suitable (problem dependent) constitutive equation relating the stresses with strains:

$$
\sigma_{i j}=W_{i j}\left(\varepsilon_{k l}\right)
$$

with a suitable strain tensor $\varepsilon_{k l}$. Here, for the considered test cases the St. VenantKirchhoff material is employed (e.g., (Ogden, 1997)).

The problem formulation has to be closed by prescribing suitable boundary and interface conditions. On solid and fluid boundaries $\Gamma_{\mathrm{s}}$ and $\Gamma_{\mathrm{f}}$ standard conditions as for individual solid and fluid problems can be prescribed. For the velocities and the stresses on a fluid-solid interface $\Gamma_{\mathrm{i}}$ we have the conditions

$$
v_{i}=\dot{u}_{i}^{\mathrm{b}} \text { and } \sigma_{i j} n_{j}=T_{i j} n_{j}
$$

where $\dot{u}_{i}^{\mathrm{b}}$ is the velocity of the interface.

\section{Numerical fluid structure coupling scheme}

The discretization of the problem domain is based on a block-structuring technique. Fluid and solid parts are assigned to different blocks. Solid blocks are treated by the finite-element solver FEAP (see (Taylor, 2002)). For the fluid blocks, which can be defined as moving or fixed, the parallel multigrid finite-volume flow solver FASTEST is employed (see (FASTEST, 2004; Schäfer et al., 2001)). Both solvers involve second-order spatial discretizations and fully implicit second-order time discretizations.

For the fluid structure coupling an implicit partitioned approach is employed. In Figure 1 a schematic view of the iteration process, which is performed for each time step, is given. After the initializations the flow field is determined in the actual flow geometry. From this the friction and pressure forces on the interacting walls are computed. These are passed to the structural solver as boundary conditions. The structural 
solver computes the deformations, with which then the fluid mesh is modified. Afterwards the flow solver is started again.

In the fluid solver a discrete form of the space conservation law

$$
\frac{\mathrm{d}}{\mathrm{d} t} \int_{V_{\mathrm{f}}} \mathrm{d} V=\int_{S_{\mathrm{f}}} v_{j}^{\mathrm{g}} n_{j} \mathrm{~d} S
$$

is taken into account in order to compute the additional connective fluxes in [1]-[2] for blocks that are moving. This is done via the swept volumes $\delta V_{c}$ of the control volume faces for which one has the relation (see (Demirdžić et al., 1998) and (Demirdžić et al., 1990)):

$$
\sum_{c} \frac{\delta V_{c}^{n}}{\Delta t_{n}}=\frac{V_{\mathrm{f}}^{n}-V_{\mathrm{f}}^{n-1}}{\Delta t_{n}}=\sum_{c}\left(v_{j}^{\mathrm{g}} n_{j} S_{\mathrm{f}}\right)_{c}^{n}
$$

where the summation index $c$ runs over the faces of the control volume, the index $n$ denotes the time level $t_{n}$ and $\Delta t_{n}$ is the time step size. By this way interface displacements enter the fluid problem part in a manner strictly ensuring mass conservation.

The fluid structure interaction (FSI) iteration loop is repeated until a convergence criterion $\varepsilon$ is reached, which is defined by the change of the mean displacements:

$$
\Re^{\mathrm{FSI}}=\max _{i=1,2,3} \frac{\sum_{k=1}^{N}\left|u_{i}^{k, m-1}-u_{i}^{k, m}\right|}{N}<\varepsilon,
$$

where $m$ is the FSI iteration counter and $N$ is the number of interface nodes. Note that an explicit coupling method would be obtained, if only one FSI iteration is performed.

The data transfer between the flow and solid solvers within the partitioned solution procedure is performed via an interface realized by the coupling library MpCCI (see (SCAI, 2004)). In Figure 2 the corresponding information flow is represented schematically. MpCCI is used for controlling the data communication as well as for carrying out the interpolations of the data from the fluid and solid grids.

After the initialization MpCCI is provided with the geometry information at the fluid-solid interface for both grids. From the flow solver these are the coordinates of the control volume vertices and centers at the interface. From the structural solver only the node coordinates are required. With these geometry informations the forces at the nodes of the structural grid are interpolated and passed to the structural solver. The displacements from the structural solver at the nodes are transfered to MpCCI, which interpolates the displacements to the control volume vertices of the fluid grid interface. Afterwards the complete fluid grid is adapted (see below) and the corresponding coordinates of the control volume centers are computed. Finally, the new geometry informations are passed to $\mathrm{MpCCI}$ for the next iteration. 


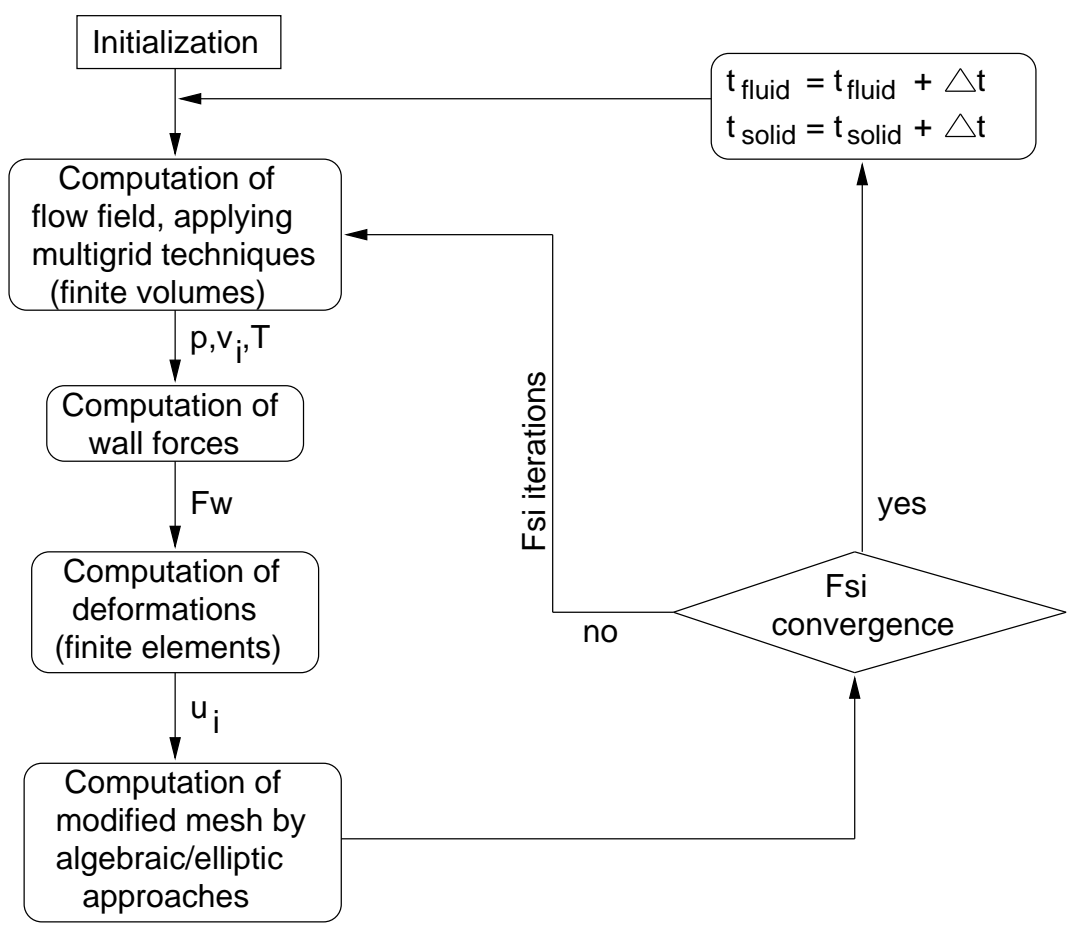

Figure 1. Flow chart of coupled solution procedure

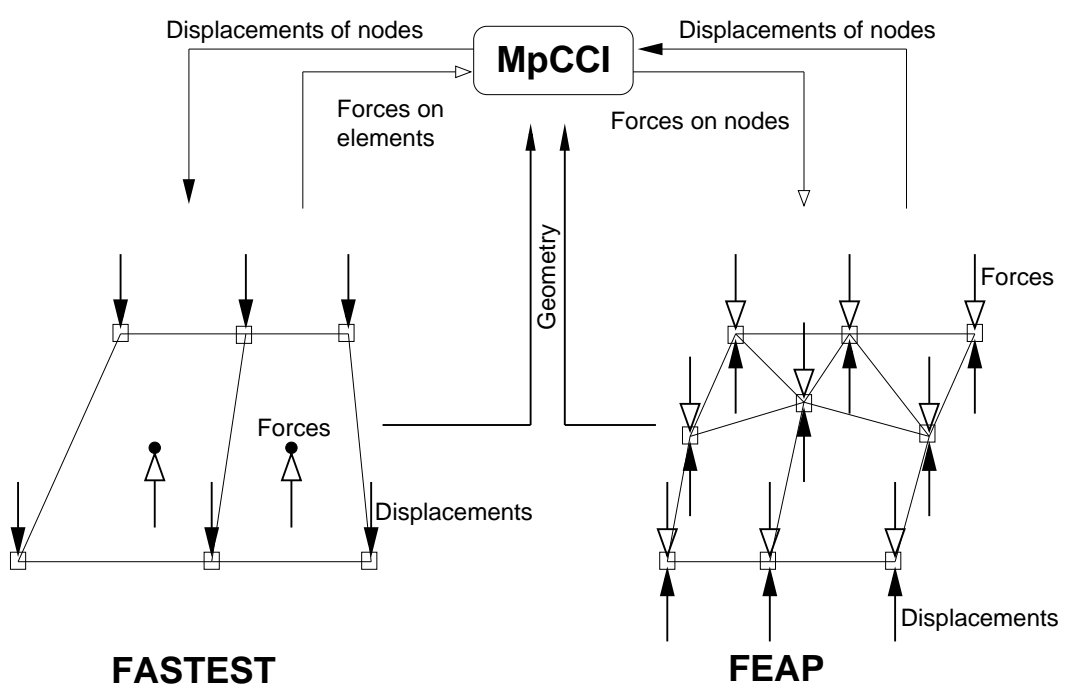

Figure 2. Schematic representation of information flow between fluid and solid solvers via the MpCCI interface 
An update of the geometry information of the solid grid is not necessary, since the structural finite-element computations always relate to the original solid grid. Note that with the considered approach nearly arbitrary discretizations for the fluid and solid subproblems can be used, i.e., there is no need for matching grids.

Various test computations have shown that the coupling scheme is rather sensitive with respect to the deformations in the first FSI iterations. Here, situations that are far away from the physical equilibrium can arise, which may lead to instabilities or even the divergence of the FSI iterations. In order to counteract this effect an underrelaxation is employed. The actually computed displacements $u^{\text {act }}$ are (linearly) weighted with the values $u_{i}^{\text {old }}$ from the preceding iteration to give the new displacements $u_{i}^{\text {new }}$ :

$$
u_{i}^{\text {new }}=\alpha_{\mathrm{FSI}} u_{i}^{\text {act }}+\left(1-\alpha_{\mathrm{FSI}}\right) u_{i}^{\text {old }},
$$

where $0<\alpha_{\text {FSI }} \leq 1$. Note that the underrelaxation does not change the final converged result.

\section{Grid movement techniques}

The method for moving the grid in the fluid domain constitutes an important component of the coupled solution procedure, in particular in the case of larger structural deformations. Besides the requirements that no grid folding occurs and that the mesh exactly fits the moving boundaries one has to take care that distortions of control volumes are kept to a minimum in order not to deteriorate the discretization accuracy and the efficiency of the solver.

\subsection{Numerical schemes}

We consider algebraic and elliptic mesh generation techniques for the grid movement with capability of boundary orthogonality. To simplify the presentation we describe the approaches for a single two-dimensional structured block surrounded by 4 boundary curves I to IV (see Figure 3). The generalization to the three-dimensional case and to multiple blocks is straightforward.

For a structured two-dimensional block there is a one-to-one mapping $\vec{x}(\xi, \eta)=$ $(x(\xi, \eta), y(\xi, \eta))$ of the physical coordinates $\vec{x}=(x, y)$ to computational coordinates $(\xi, \eta)$ where without loss of generality $0 \leq \xi \leq 1$ and $0 \leq \eta \leq 1$. In each FSI iteration the coordinates of the interior grid points have to be computed from the (given) boundary points distribution. 

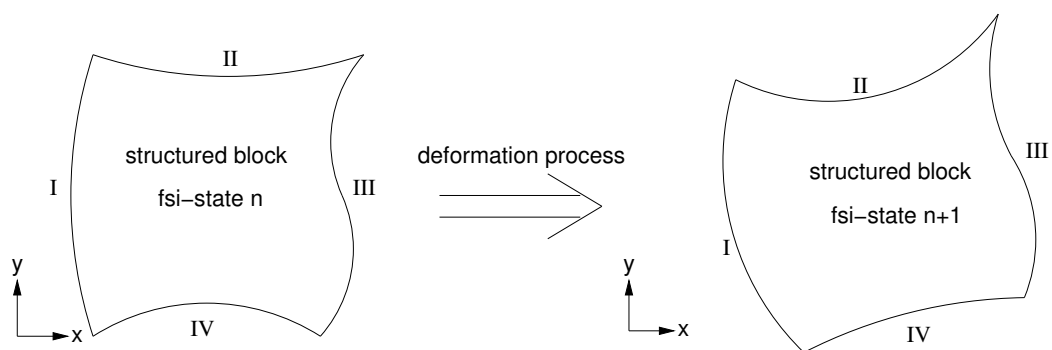

Figure 3. Deformation of structured two-dimensional block

First, we consider algebraic approaches. A very simple method is obtained by linear interpolation between opposite boundaries, e.g. boundary I to III. Let the distances between neighboring grid points be

$$
d_{i, j}=\left\|\vec{x}_{i, j}-\vec{x}_{i-1, j}\right\| \quad i=1 \ldots N, j=0 \ldots M
$$

with the overall lengths

$$
L_{j}=\sum_{i=1}^{N} d_{i, j} \quad j=0 \ldots M
$$

The normalized lengths for the parametrization are

$$
\bar{L}_{i, j}=\frac{1}{L_{j}} \sum_{m=1}^{i} d_{m, j} \quad i=1 \ldots N, j=0 \ldots M, \bar{L}_{0, j}=0
$$

and finally the whole domain is computed by

$$
\vec{x}_{i, j}=\left(\vec{x}_{N, j}-\vec{x}_{0, j}\right) \cdot \bar{L}_{i, j}+\vec{x}_{0, j} \quad i=0 \ldots N, j=0 \ldots M
$$

A further algebraic method is the linear transfinite interpolation (TFI), where the interior grid points are computed by

$$
\begin{aligned}
\vec{x}(\xi, \eta)= & (1-\eta) \vec{x}(\xi, 0)+\eta \vec{x}(\xi, 1)+(1-\xi) \vec{x}(0, \eta)+\xi \vec{x}(1, \eta) \\
& -\xi[\eta \vec{x}(1,1)+(1-\eta) \vec{x}(1,0)] \\
& -(1-\xi)[\eta \vec{x}(0,1)+(1-\eta) \vec{x}(0,0)] .
\end{aligned}
$$

The algebraic methods are simple, but deteriorated grid cells and even grid folding may occur. 
For an elliptic grid movement we adopt an approach described in (Thompson et $a l ., 1999)$ which is based on the following (elliptic) Poisson equation for the physical coordinates:

$$
\begin{aligned}
a \vec{x}_{\xi \xi}-2 b \vec{x}_{\xi \eta}+c \vec{x}_{\eta \eta} & +\left(a P_{11}^{1}-2 b P_{12}^{1}+c P_{13}^{1}\right) \vec{x}_{\xi} \\
& +\left(a P_{11}^{2}-2 b P_{12}^{2}+c P_{13}^{2}\right) \vec{x}_{\eta}=\overrightarrow{0}
\end{aligned}
$$

with the control functions

$$
\begin{aligned}
\vec{P}_{11} & =\frac{1}{s_{\eta} t_{\xi}-s_{\xi} t_{\eta}}\left[\begin{array}{cc}
t_{\eta} & -s_{\eta} \\
-t_{\xi} & s_{\xi}
\end{array}\right]\left[\begin{array}{c}
s_{\xi \xi} \\
t_{\xi \xi}
\end{array}\right] \\
\vec{P}_{12} & =\frac{1}{s_{\eta} t_{\xi}-s_{\xi} t_{\eta}}\left[\begin{array}{cc}
t_{\eta} & -s_{\eta} \\
-t_{\xi} & s_{\xi}
\end{array}\right]\left[\begin{array}{l}
s_{\xi \eta} \\
t_{\xi \eta}
\end{array}\right] \\
\vec{P}_{13} & =\frac{1}{s_{\eta} t_{\xi}-s_{\xi} t_{\eta}}\left[\begin{array}{cc}
t_{\eta} & -s_{\eta} \\
-t_{\xi} & s_{\xi}
\end{array}\right]\left[\begin{array}{c}
s_{\eta \eta} \\
t_{\eta \eta}
\end{array}\right]
\end{aligned}
$$

and the abbreviations

$$
a=x_{\eta} x_{\eta}+y_{\eta} y_{\eta}, \quad b=x_{\xi} x_{\eta}+y_{\xi} y_{\eta}, \quad c=x_{\xi} x_{\xi}+y_{\xi} y_{\xi}
$$

An index $\xi$ and $\eta$ denotes the corresponding derivatives. Figure 4 shows how the mapping between the computational space and the physical domain is performed through the parameter space $(s, t)$ that can be used to control the quality of the mesh.

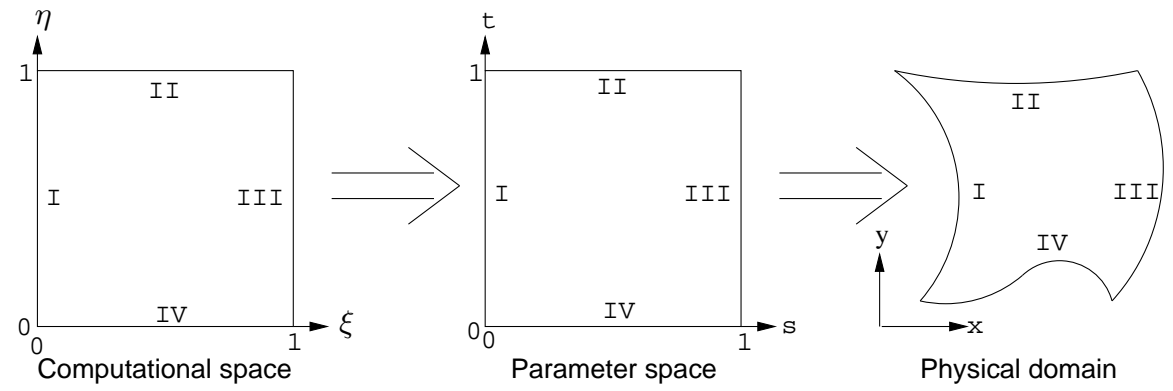

Figure 4. Mapping strategy for elliptic grid movement

We consider a parameter space with $s(\mathrm{I})=0, s(\mathrm{III})=1, t(\mathrm{IV})=0$, and $t(\mathrm{II})=1$. The point distribution along $s(\mathrm{II}), s(\mathrm{IV}), t(\mathrm{I})$, and $t(\mathrm{III})$ arises from linear interpolation along these boundaries involving the normalized arc length. The inner parameter domain is adapted by solving simultaneously:

$$
\begin{aligned}
s & =s(\mathrm{IV})(1-t)+s(\mathrm{II}) t \\
t & =t(\mathrm{I})(1-s)+t(\mathrm{III}) s .
\end{aligned}
$$


Next, the control functions $P_{11}^{i}, P_{12}^{i}, P_{13}^{i}, i=1,2$ can be computed according to [17] to [19] and remain unchanged during the solution of [16]. The derivatives in Equation [16] are approximated by central differences and a Picard iteration process is used for linearization:

$$
\begin{gathered}
a^{k-1} \vec{x}_{\xi \xi}^{k}-2 b^{k-1} \vec{x}_{\xi \eta}^{k}+c^{k-1} \vec{x}_{\eta \eta}^{k} \\
+\left(a^{k-1} P_{11}^{1}-2 b^{k-1} P_{12}^{1}+c^{k-1} P_{13}^{1}\right) \vec{x}_{\xi}^{k} \\
+\left(a^{k-1} P_{11}^{2}-2 b^{k-1} P_{12}^{2}+c^{k-1} P_{13}^{2}\right) \vec{x}_{\eta}^{k}=\overrightarrow{0} .
\end{gathered}
$$

In each step this equation system is solved by the Gauß-Seidel algorithm (the computation time for this step is negligible compared to the total time) for the unknown grid coordinates $x_{i, j}^{k}$ and $y_{i, j}^{k}, i=1 \ldots N-1, j=1 \ldots M-1$. The Picard iteration process is repeated as long as a certain convergence criterion is satisfied. Within the FSI iteration process the coordinates of the previous iteration are applied as initial values. The whole solution algorithm, which is summarized schematically in Figure 5 operates like a mesh smoother. In any case a boundary conforming mesh without grid folding results.

The described method can be extended according to (Thompson et al., 1999), (Spekreijse, 1995) with respect to boundary orthogonality. First a boundary conforming grid without grid folding is computed with the elliptic method explained above. On this mesh we consider the Laplace equations:

$$
\begin{aligned}
\triangle s & =\frac{\partial^{2} s}{\partial x^{2}}+\frac{\partial^{2} s}{\partial y^{2}}=\left(\frac{1}{J} a s_{\xi}-\frac{1}{J} b s_{\eta}\right)_{\xi}+\left(-\frac{1}{J} b s_{\xi}+\frac{1}{J} c s_{\eta}\right)_{\eta}=0 \\
\Delta t & =\frac{\partial^{2} t}{\partial x^{2}}+\frac{\partial^{2} t}{\partial y^{2}}=\left(\frac{1}{J} a t_{\xi}-\frac{1}{J} b t_{\eta}\right)_{\xi}+\left(-\frac{1}{J} b t_{\xi}+\frac{1}{J} c t_{\eta}\right)_{\eta}=0
\end{aligned}
$$

with the abbreviations

$$
\begin{aligned}
a=x_{\eta} x_{\eta}+y_{\eta} y_{\eta}, \quad b=x_{\xi} x_{\eta}+y_{\xi} y_{\eta}, \\
c=x_{\xi} x_{\xi}+y_{\xi} y_{\xi}, \quad J=x_{\xi} y_{\eta}-x_{\eta} y_{\xi},
\end{aligned}
$$

in combination with the Neumann boundary conditions

$$
\frac{\partial s}{\partial \vec{n}}=0, \quad \frac{\partial t}{\partial \vec{n}}=0,
$$




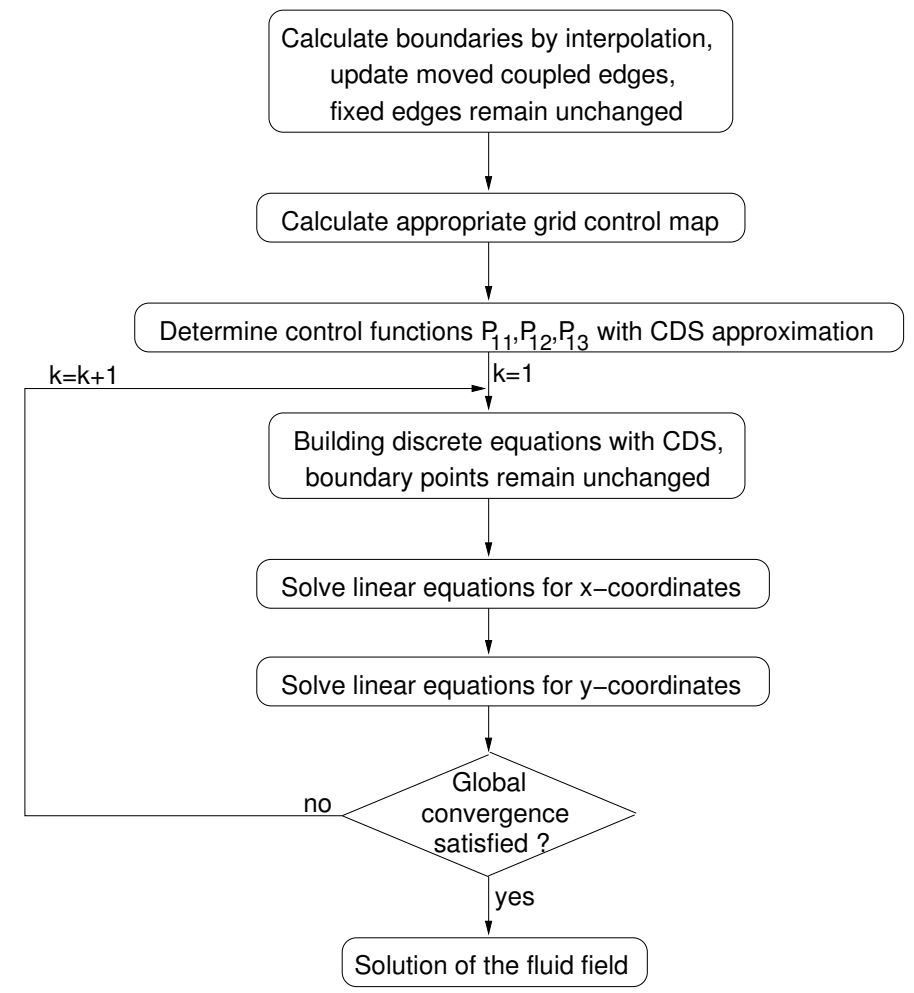

Figure 5. Flow chart of elliptic mesh movement method

where $\vec{n}=\left(n_{1}, n_{2}\right)$ is the outward unit normal vector. Since the calculations for both variables $s$ and $t$ are similar, further derivations are outlined for $s$ only. Equation [24] involves a divergence expression that allows for applying the finite volume method:

$$
\begin{aligned}
& \int_{\Omega}\left(\frac{1}{J} a s_{\xi}-\frac{1}{J} b s_{\eta}\right)_{\xi}+\left(-\frac{1}{J} b s_{\xi}+\frac{1}{J} c s_{\eta}\right)_{\eta} d \xi d \eta \\
& =\int_{\partial \Omega}\left[\frac{1}{J}\left(s_{\xi}\left(a n_{1}-b n_{2}\right)+s_{\eta}\left(-b n_{1}+c n_{2}\right)\right)\right] d \sigma=0,
\end{aligned}
$$

where the integration is done for a control volume $\Omega$ and its boundary $\partial \Omega$ with the line element $d \sigma$, respectively. The computational domain is discretized by unit control volumes for inner points and half control volumes for boundary points, leading to one system of linear equations for $s$. Since the boundary condition [27] transforms to

$$
\frac{1}{J}\left(s_{\xi}\left(a n_{1}-b n_{2}\right)+s_{\eta}\left(-b n_{1}+c n_{2}\right)\right)=0
$$


these terms have to be set to zero for the desired orthogonal grid lines at boundaries. During the solution process, boundary points are moved along edges $(s(I V), s(I I))$ until convergence is reached. These points are combined by cubic Hermite interpolation:

$$
\begin{array}{ll}
s=s(I V)(1+2 t)(1-t)^{2}+s(I I)(3-2 t) t^{2}, & 0 \leq t \leq 1 \\
t=t(I)(1+2 s)(1-s)^{2}+t(I I I)(3-2 s) s^{2}, & 0 \leq s \leq 1
\end{array}
$$

Since the interpolation is analytically given, the Jacobian matrix and its inverse can easily be calculated and solved simultaneously for $s$ and $t$ by the Newton algorithm. As initial condition the parameter space values from the first elliptic solution are applied, leading to convergence after one or two Newton iterations. Finally the desired grid is computed by solving the elliptic equations once again with the new control functions. Figure 6 shows the whole algorithm schematically on the basis of an asymmetrically deformed domain with boundary orthogonality on all four edges.

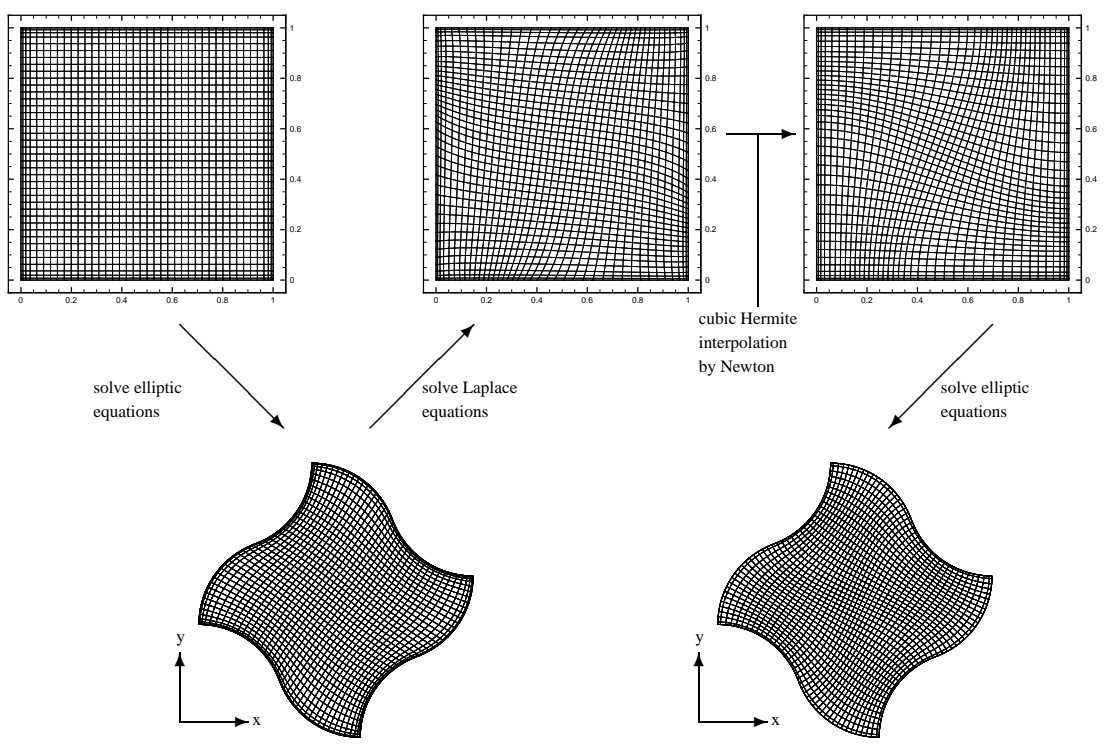

Figure 6. Example of generating a boundary orthogonal grid

The advantages of this approach lie in the great flexibility allowing independent orthogonalization of all four boundaries while keeping the edge point distribution unchanged. Furthermore the method is very robust and even works in cases of huge deformations (see Section 4.2). 


\subsection{Numerical results}

In this section, we investigate the influence of the grid movement and compare the algebraic and elliptic approaches described in Section 4.1. As a test case we consider a laminar flow around a rotating cylinder fixed in a channel which allows for a systematic variation of the amount of grid movement. Figure 7 shows the geometry and the corresponding block structure consisting of two fixed blocks at the inlet and the outlet and four moving blocks belonging to an $\mathrm{O}$-grid around the cylinder. The kinematic viscosity is defined as $\nu_{\mathrm{f}}=10^{-3} \mathrm{~m}^{2} / \mathrm{s}$ and the fluid density is $\rho_{\mathrm{f}}=1.0 \mathrm{~kg} / \mathrm{m}^{3}$. At the inflow a parabolic velocity profile with mean velocity $\bar{u}=0.2 \mathrm{~m} / \mathrm{s}$ which together with the cylinder diameter of $D=0.1 \mathrm{~m}$ gives the Reynolds number $R e=20$. To get a physically two-dimensional problem a symmetry boundary condition is applied in the third direction. The cylinder is rotated in steps of 5 degrees. The grid adaption according to the rotating cylinder is done on the one hand by linear and transfinite interpolation belonging to algebraic methods and on the other hand by elliptic approaches also supplying boundary orthogonality as outlined in Section 4.1. After each step a steady flow computation is performed until grid folding or divergence of the iterations occur. 125000 control volumes and exactly the same parameters are used for all cases and for all angles of torsion. For comparison a reference solution obtained by a computation with 450000 control volumes on an undeformed mesh is considered.

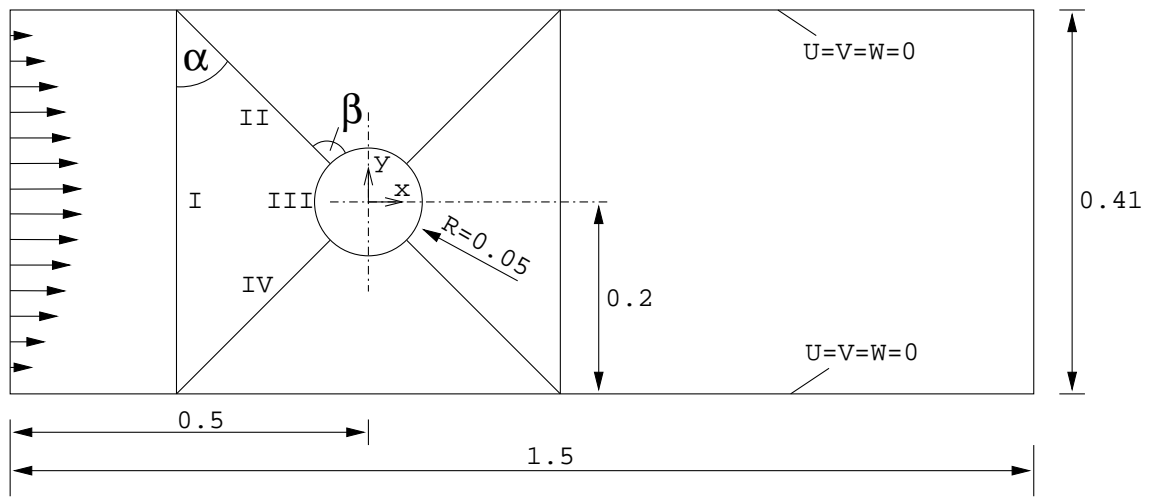

Figure 7. Test configuration for grid movement techniques (units in m)

In Figures 8 and 9 the grids with the maximum angle of torsion for which the last converged solution could be achieved are illustrated. It should be mentioned that, except the linear interpolation, the boundaries II and IV are approximated by a cubic spline interpolation to get angles of about $\alpha=45^{\circ}$ and $\beta=90^{\circ}$ (see Figure 7). While the elliptic approach allows for an angle of $95^{\circ}$ the linear interpolation works until $70^{\circ}$. TFI begins to fail from $50^{\circ}$ on. The elliptic approach in combination with boundary orthogonality reaches values even up to $100^{\circ}$.

In Figure 10 a comparison of the lift coefficients with varying rotation angle is shown. The TFI is nearly congruent with the orthogonal elliptic approach that also 
gives satisfying results even for larger angles. The usual elliptic method shows nearly the same values. The linear interpolation leads to high deviations from begin on.
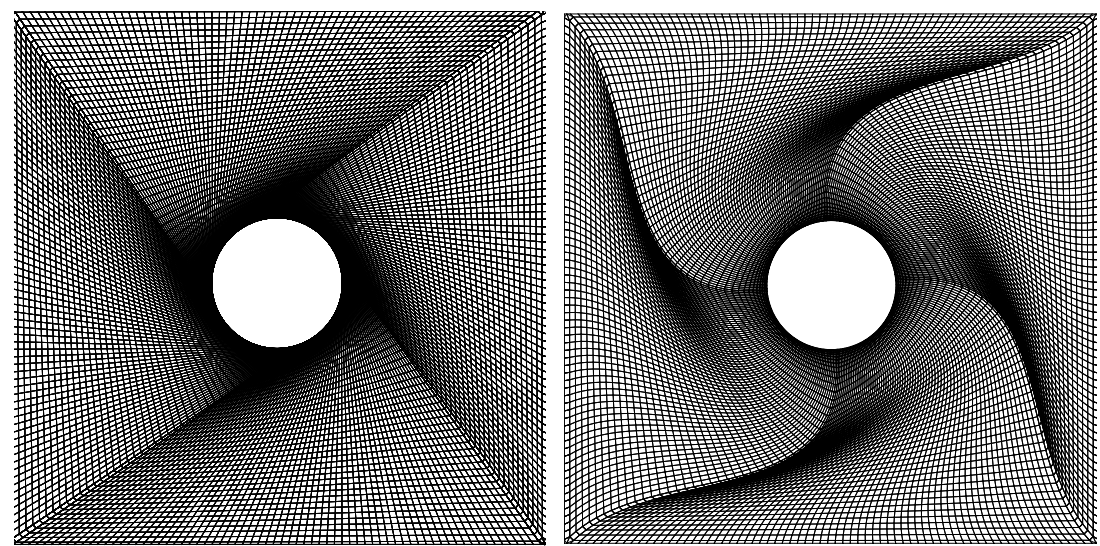

Figure 8. Maximum possible grid distortions with linear (left, $\left.70^{\circ}\right)$ and transfinite interpolated (right, $\left.50^{\circ}\right)$ grid movements
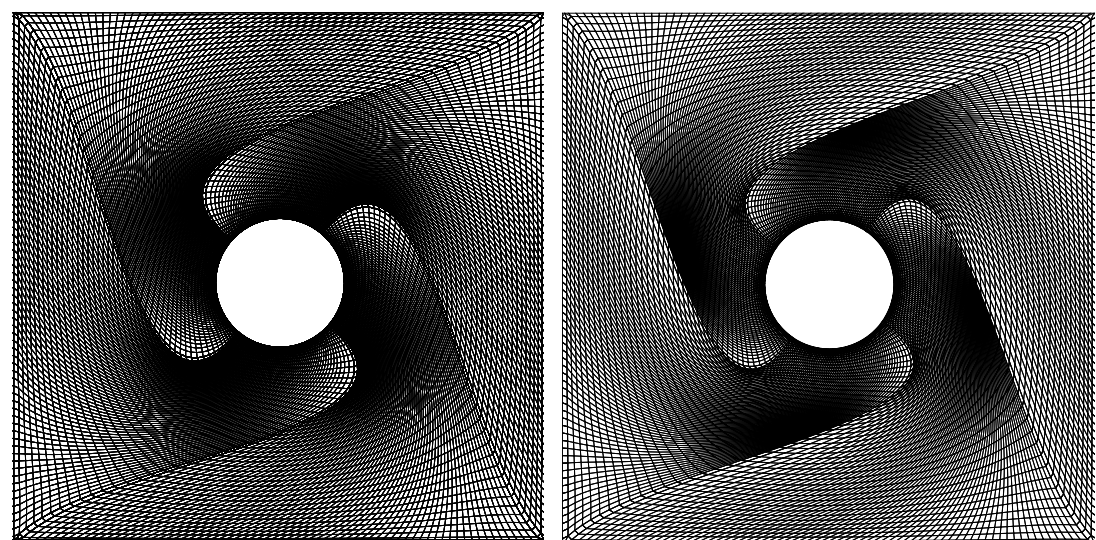

Figure 9. Maximum possible grid distortions with elliptic smoother (left, 95\%) and additional boundary orthogonality around the cylinder $\left(\right.$ right, $\left.100^{\circ}\right)$

In Figure 11 the numbers of iterations required to obtain the converged solution for varying rotation angle are indicated. The iteration numbers increase with increasing angle. While for small angles the linear interpolation is slightly faster, the situation changes for larger angles due to the heavy distortions of control volumes near the boundary. The values for TFI increase dramatically caused by grid distortions around the cubic spline.

In summary, considering both, accuracy and efficiency, one can state that for small distortions, i.e., with angles of torsion $\approx 0^{\circ}-20^{\circ}$, the algebraic methods work satis- 
factorily, but for larger angles with the elliptic approaches significantly more accurate results can be obtained. The latter even still work in cases where the algebraic approaches leads to grid folding. TFI only is useful for very small displacements because of the high computing times for larger deformations.

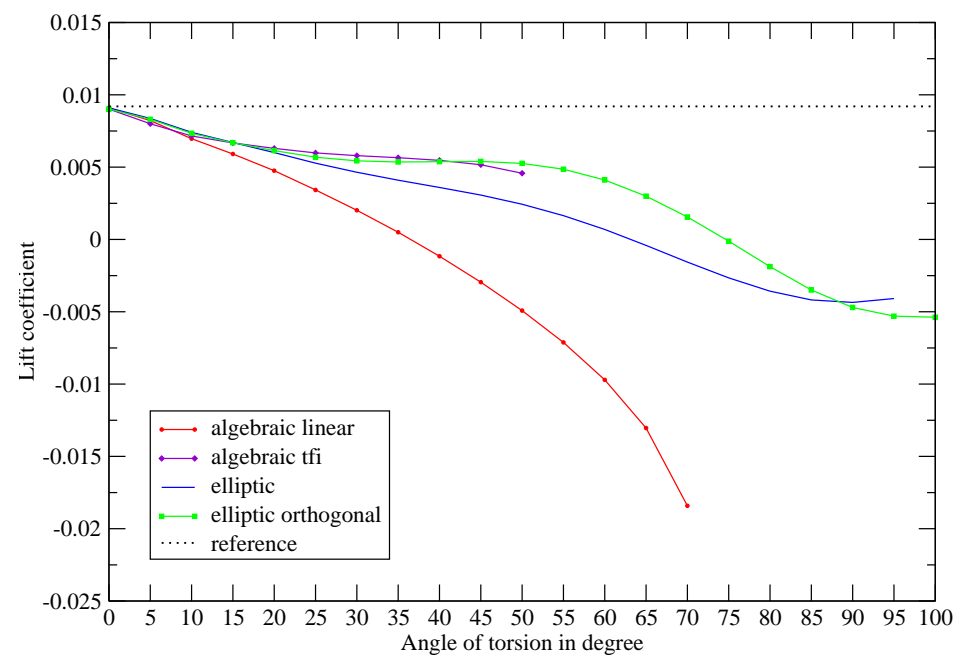

Figure 10. Comparison of lift coefficients with algebraic and elliptic grid movements

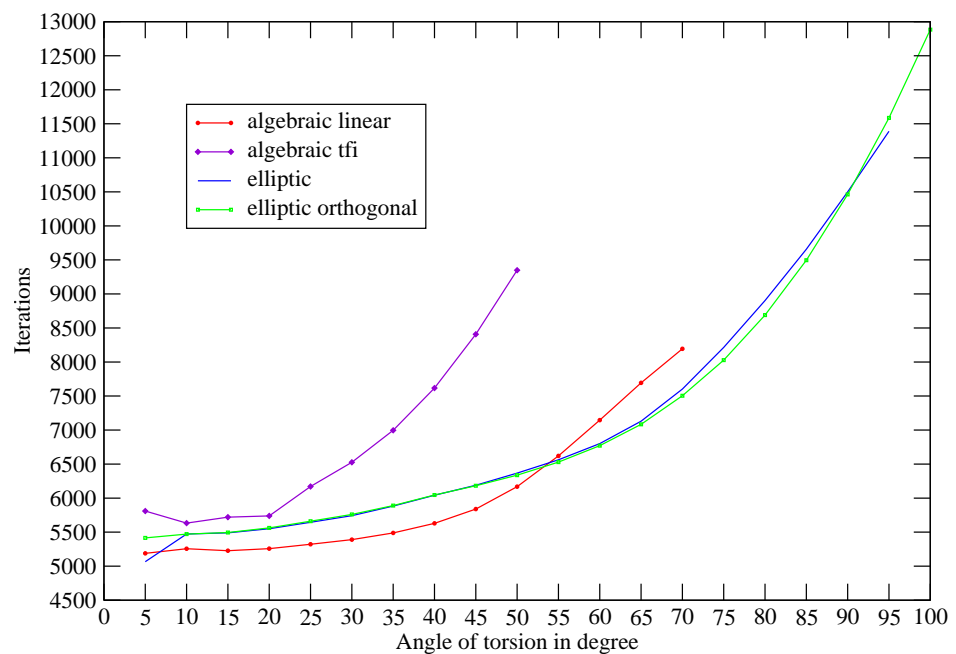

Figure 11. Comparison of computational effort with algebraic and elliptic grid movements 


\section{Numerical investigation of fluid excited vibrations}

\subsection{Comparison with experiments}

First, fluid structure interaction phenomena are studied on a real test case. The corresponding experiments are performed by the Institute of Fluid Mechanics at University of Erlangen-Nürnberg (see (Gomes et al., 2006)).

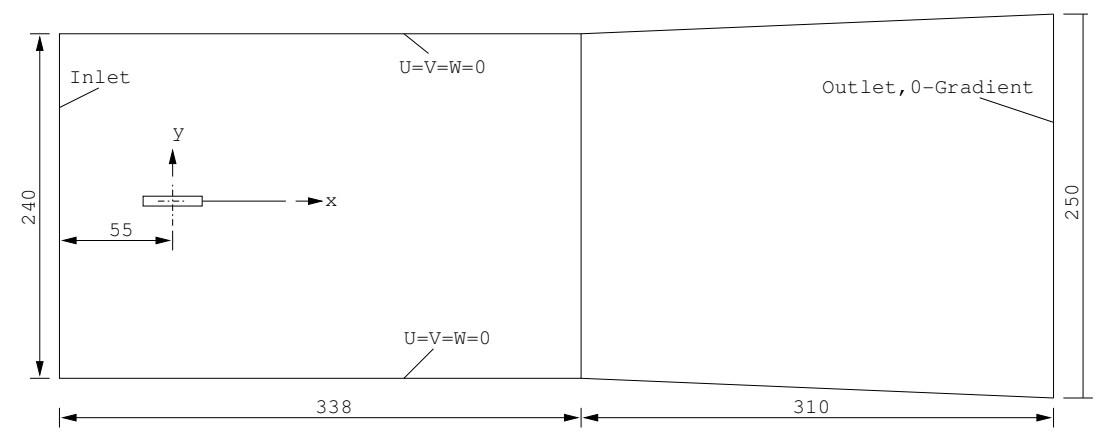

Figure 12. Geometric properties of test channel (units in $\mathrm{mm}$ )

Figure 12 shows the geometric properties of the test channel including the structural configuration presented in Figure 13. For the (highly viscous) fluid polyethylene glycol is chosen with density $\rho_{\mathrm{f}}=1050 \mathrm{~kg} / \mathrm{m}^{3}$ and kinematic viscosity $\nu_{\mathrm{f}}=$ $1.64 \cdot 10^{-4} \mathrm{~m}^{2} / \mathrm{s}$. The structural configuration consists of a cylindrical aluminum front body $\left(\rho_{\mathrm{s}}=2828 \mathrm{~kg} / \mathrm{m}^{3}, E=7 \cdot 10^{10} \mathrm{~N} / \mathrm{m}^{2}\right)$, a thin membrane of stainless steel $\left(\rho_{\mathrm{s}}=7855 \mathrm{~kg} / \mathrm{m}^{3}, E=2 \cdot 10^{11} \mathrm{~N} / \mathrm{m}^{2}\right)$, and a rectangular rear mass $\left(\rho_{\mathrm{s}}=\right.$ $\left.7800 \mathrm{~kg} / \mathrm{m}^{3}, E=2 \cdot 10^{11} \mathrm{~N} / \mathrm{m}^{2}\right)$. The configuration is fixed in the channel with one rotational degree of freedom positioned at the center of the cylinder, where no friction is assumed at the fixing point. The gravity force is aligned with the $x$-axis. The physical problem can be considered as two-dimensional what was confirmed by several measurements. In the simulations this is represented by symmetry boundary conditions in $z$-direction.

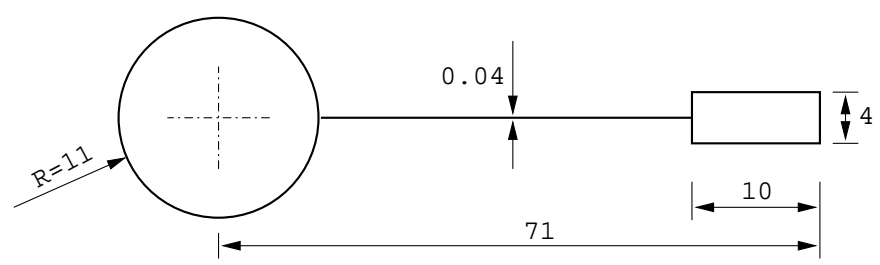

Figure 13. Sketch of structural configuration 
For the structural discretization 920 linear solid hexahedrons (in-plane) are applied in combination with enhanced strain formulations (see (Taylor, 2003)) allowing for very large aspect ratios. The fluid domain is discretized by 13444 (in-plane) control volumes. For mesh adaption the elliptic method is applied.

For a better understanding of the fluid structure interaction mechanism first an eigenfrequency analysis for the structure is carried out by a pure structural simulation. The first two eigenmodes are shown in Figure 14 (the trivial rigid body motion with $0.048 \mathrm{~Hz}$ is not considered). In the first mode with frequency $6.12 \mathrm{~Hz}$ the front body moves in the same direction as the rear mass, while in the second mode with frequency $30.07 \mathrm{~Hz}$ the moving directions of the front and the end mass are opposite.

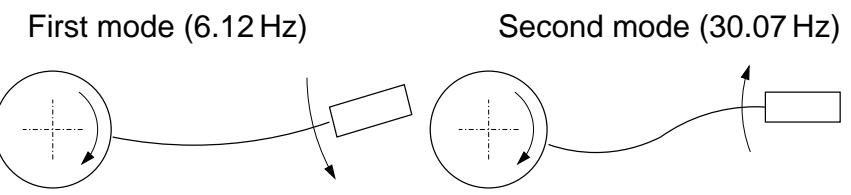

Figure 14. Eigenmodes of structural configuration

\subsubsection{First swiveling motion with low inlet velocity}

Applying an uniform inlet velocity of $\bar{u}=1.14 \mathrm{~m} / \mathrm{s}(R e \approx 130)$ the structure starts vibrating by itself, in the computation as well as in the experiment, and oscillates with the first swiveling motion, i.e., the front body moves in the same direction as the rear mass. Figure 15 shows a comparison of the superposition of structural deformations. The corresponding trailing edge displacements for one period are presented in Figure 16 (experimental results from internal communication with (Gomes et al., 2006)). The slope shapes are similar. The amplitude of the computation is a little larger then in the experiment. The reason of this appears to be the assumption of a totally twodimensional problem. In the experiment a small gap between the rear mass and the side walls arises that results in a small damping effect. Since the first swiveling motion is qualitatively similar to linear small vibrations, one can state that with damping the amplitude decreases. The computed swiveling motion frequency is $7.0 \mathrm{~Hz}$ (see Figure 17) and the corresponding experimental value is $6.64 \mathrm{~Hz}$ (from internal communication with (Gomes et al., 2006)). A snapshot of the velocity component in $x$-direction is given in Figure 18. 

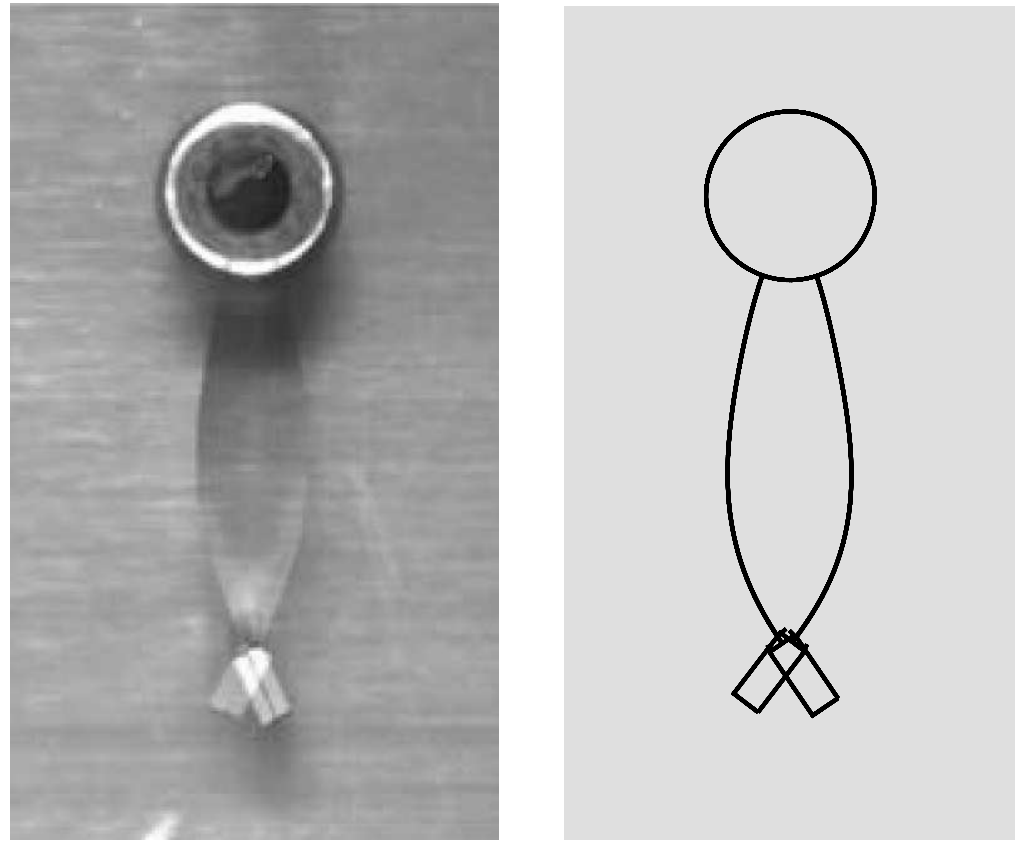

Figure 15. Superposition of images at a time-phase angle of $90^{\circ}$ and $270^{\circ}$, experiment (left), computation (right), first swiveling motion

Experiment

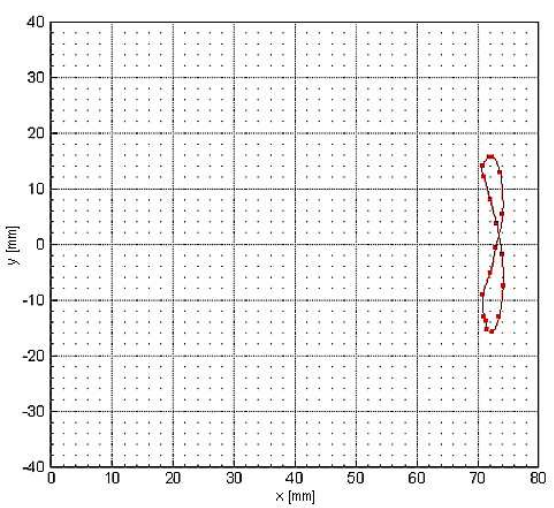

Computation

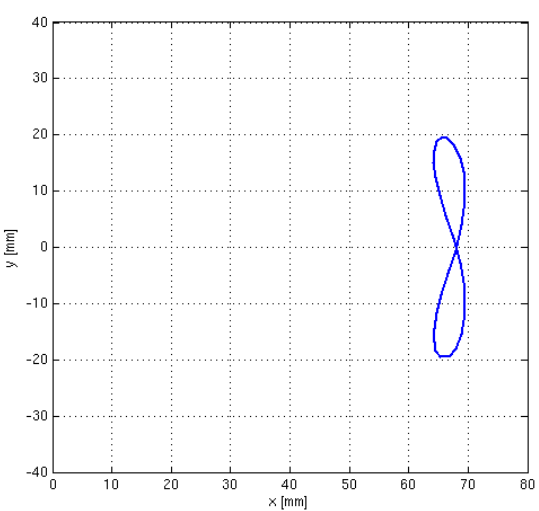

Figure 16. Comparison of trailing edge displacements for one period, first swiveling motion 


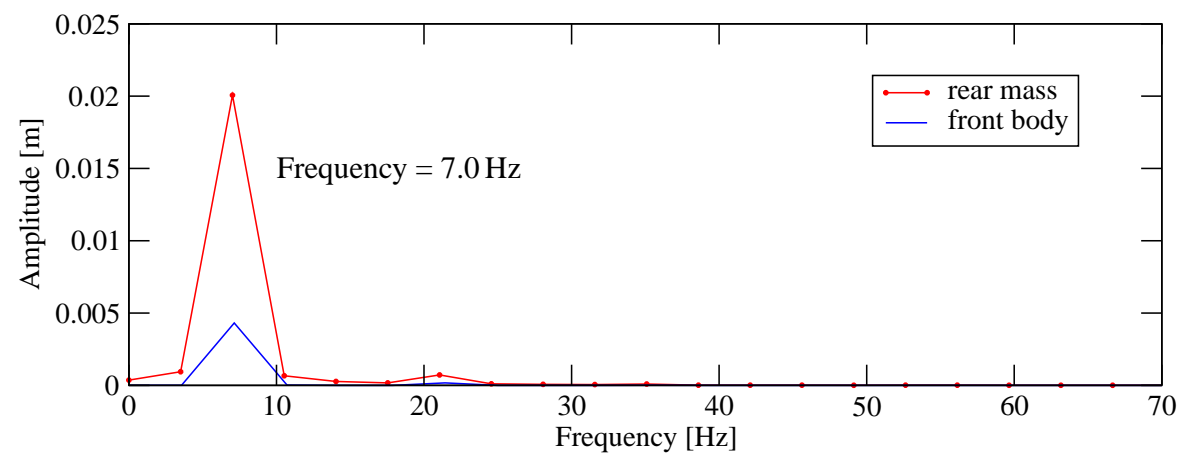

Figure 17. Spectral representation of rear mass and front body oscillations (from simulation results) for first swiveling fluid structure interaction motion

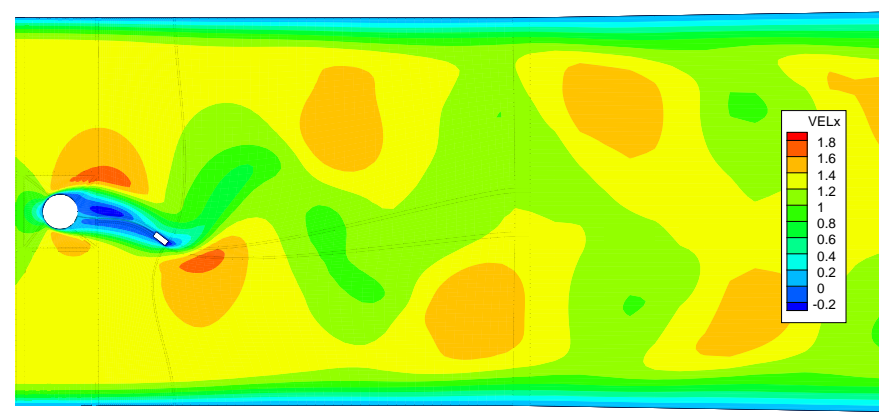

Figure 18. Snapshot of velocity in $x$-direction for first swiveling fluid structure interaction motion

\subsubsection{Second swiveling motion with higher inlet velocity}

Increasing the uniform inlet velocity to $\bar{u}=1.37 \mathrm{~m} / \mathrm{s}(R e \approx 170)$ the structure oscillates with the second swiveling motion, i.e., the front body moves in opposite direction as the rear mass. Figure 19 shows the superposition of structural deformations for experiment and computation. A comparison with Figure 15 shows the difference between the two modes, particularly the intersection point in the second case. In Figure 20 a comparison between experiment and simulation for the displacement of the trailing edge in the $x-y$-plane is shown. The experimental and numerical results are in very good agreement. The maximum $y$-amplitude in both cases is between 18 and $20 \mathrm{~mm}$. The slope shapes are nearly equal. In that case the damping effect mentioned before obviously is less significant. The experimental and numerical frequencies are $13 \mathrm{~Hz} \pm 1.5 \%$ and $12.7 \mathrm{~Hz}$, i.e., there is a very good agreement. The spectral representation of the rear mass and front body oscillations obtained from the simulation results 

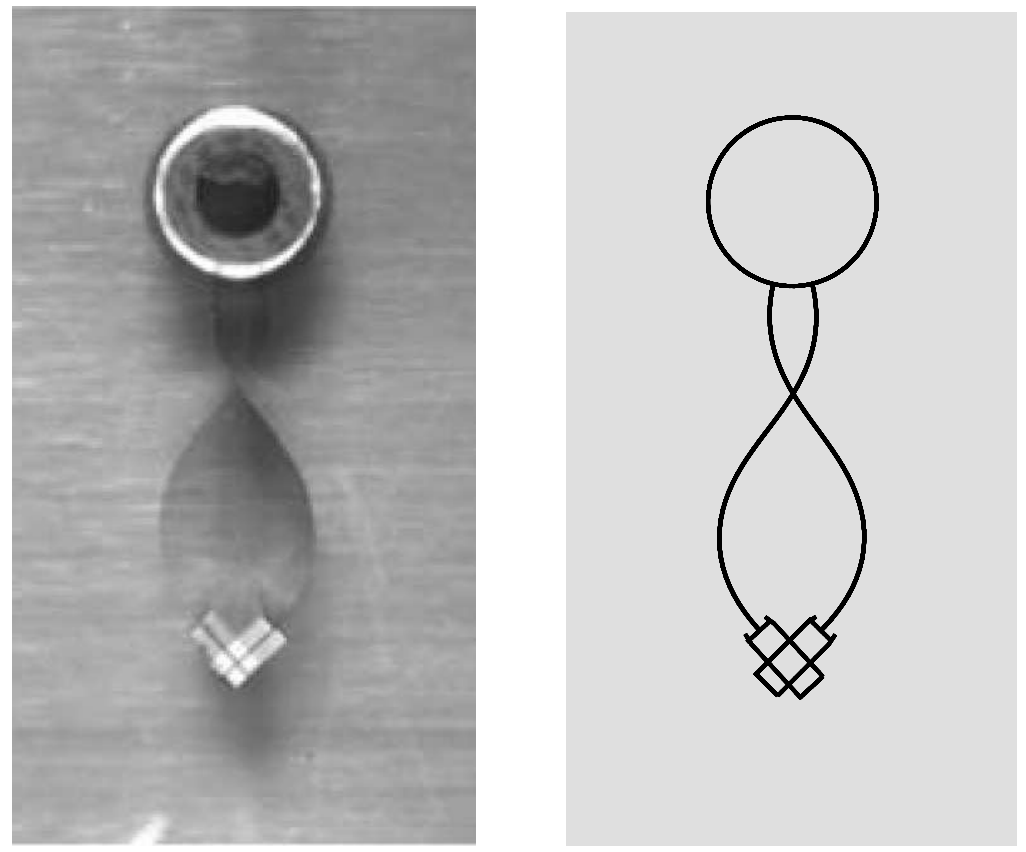

Figure 19. Superposition of images at a time-phase angle of $90^{\circ}$ and $270^{\circ}$, experiment (left), computation (right), second swiveling motion
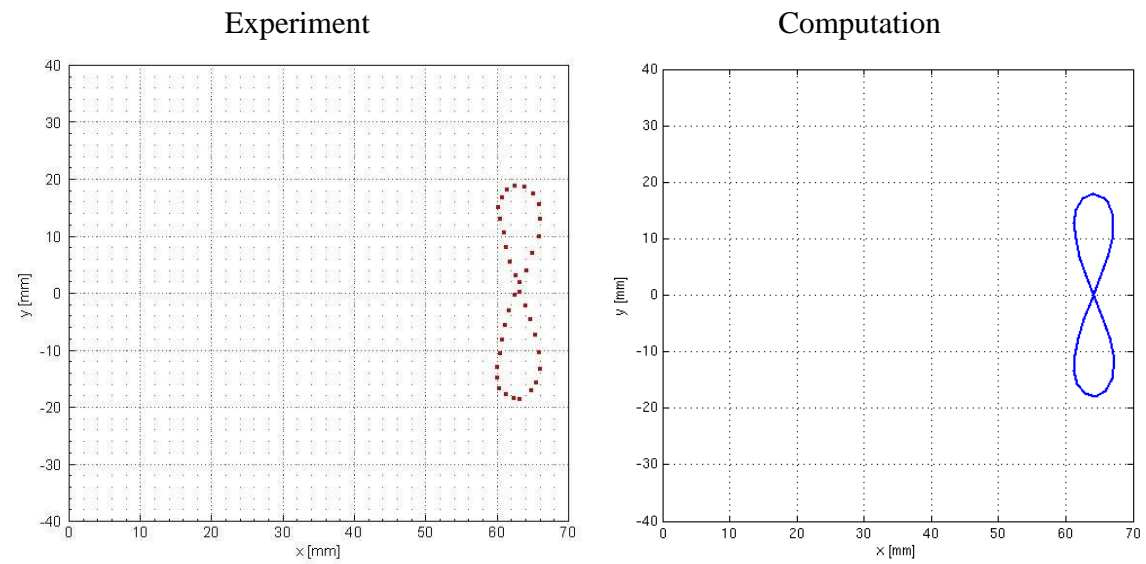

Figure 20. Comparison of trailing edge displacements for one period, second swiveling motion 
is shown in Figure 21 indicating that other occurring frequencies are negligibly small. The frequency analysis also indicates that the first swiveling motion frequency is close to the pure structural first eigenfrequency, but regarding the second swiveling motion this is not the case.

A snapshot of the velocity component in $x$-direction is given in Figure 22. The oscillation is periodic but not harmonic what is expected for non-linear vibrations. A comparison with Figure 18 illustrates the larger deformation, the higher velocities and the larger frequency in case of the second swiveling motion.

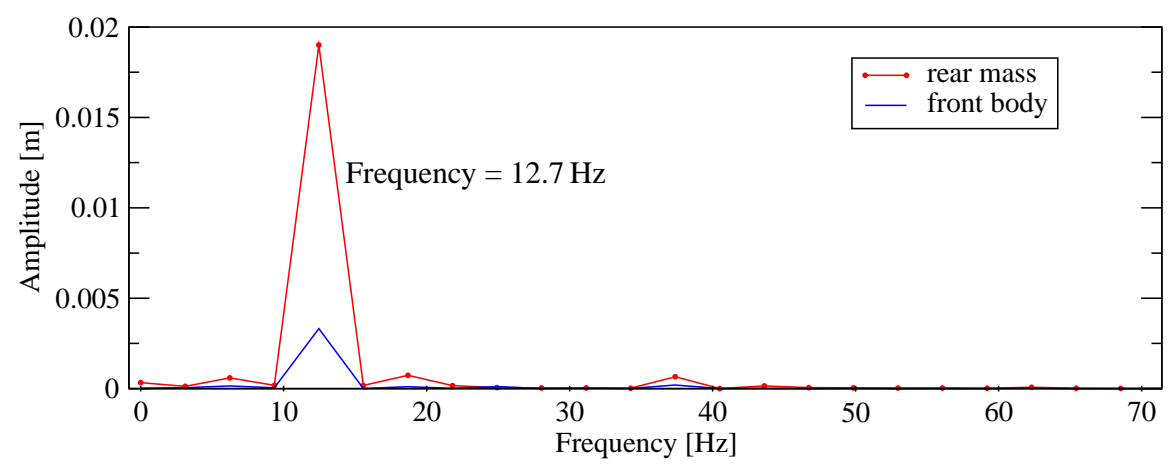

Figure 21. Spectral representation of rear mass and front body oscillations (from simulation results) for second swiveling fluid structure interaction motion

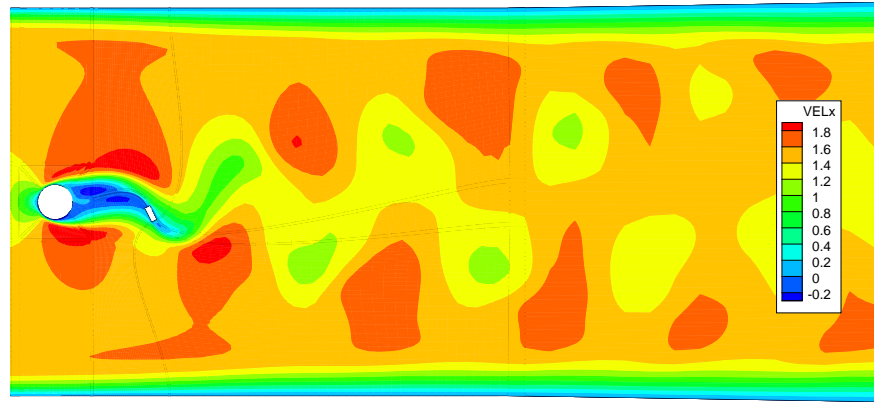

Figure 22. Snapshot of velocity in $x$-direction for second swiveling fluid structure interaction motion 


\subsection{Influence of material parameters on fluid excited structural vibrations}

Now, material properties causing different structural behavior are varied and studied systematically. The investigations are performed with regard to realistic material properties to have the possibility to compare the phenomena with those of the preceding section.

The same test channel as before is used (Figure 12) with the configurations presented in Figure 23. The structure consists of a rigid front plate $\left(\rho_{\mathrm{s}}=7855 \mathrm{~kg} / \mathrm{m}^{3}\right)$, fixed with one rotational degree of freedom in the center and an attached membrane (configuration (a)). Combining the membrane with a rear mass (geometry is fixed) at the trailing edge leads to a second configuration (configuration (b)). To limit the amount of varying parameters, both the front body and the fluid properties are kept unchanged during the investigation. For comparison the displacements of points $\mathrm{A}$ and $\mathrm{B}$, respectively, and the overall deformation are considered. As initial condition $15 \mathrm{~mm}$ deviation of point $\mathrm{B}$ is prescribed.

Configuration (a)

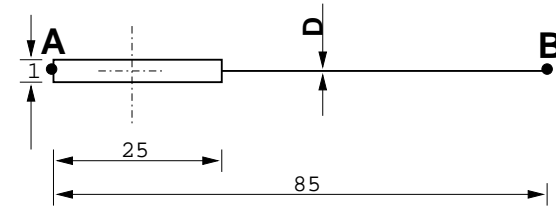

Configuration (b)

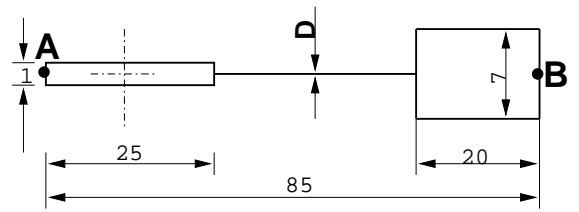

Figure 23. Structural test configurations (units in $\mathrm{mm}$ )

The fluid domain is discretized with 15840 control volumes (in-plane) and symmetry boundary conditions. For acceleration a multigrid solution approach is employed. The highly viscous fluid polyethylene glycol $\left(\rho_{\mathrm{f}}=1075 \mathrm{~kg} / \mathrm{m}^{3}\right.$, $\nu_{\mathrm{f}}=5 \cdot 10^{-4} \mathrm{~m}^{2} / \mathrm{s}$ ) with an uniform inlet velocity of $\bar{u}=2 \mathrm{~m} / \mathrm{s}$ results in a Reynolds number of $R e=340$. Since the membrane undergoes very large deformations (local strains are still moderate) the elliptic smoother is indispensable for mesh adaption, otherwise grid folding occurs. The structure is discretized by 320 linear solid hexahedrons (in-plane) applying an enhanced strain formulation. St. Venant-Kirchhoff's material law is chosen for linear material behavior with kinematics of large deformations and small strains.

All parameters and results are summarized in Table 1. First, configuration (a) is considered with real properties for the membrane $\left(\rho_{\mathrm{s}}=7855 \mathrm{~kg} / \mathrm{m}^{3}, E=2 \cdot 10^{11} \mathrm{~N} / \mathrm{m}^{2}\right)$ varying the thickness. Starting with $D=0.03 \mathrm{~mm}$ results in a non-periodic oscillation as described in Figure 24. Two images are superposed where the front plate reaches the upper and lower turning point. The front body swivels non-periodically around an angle $\alpha$. Taking Figure 25 into account, at a time of about 0.45 seconds $\alpha$ changes its sign and the swiveling motion takes place at the opposite side of the x-axis. The same effect recurs approximately at $t=0.8$ seconds. The reason for the non-periodic 
behavior is the very thin membrane. As the thickness gets smaller and smaller the structure loses its stiffness and the configuration reacts like a flag fixed in a fluid stream, i.e., the movement gets more and more chaotic.

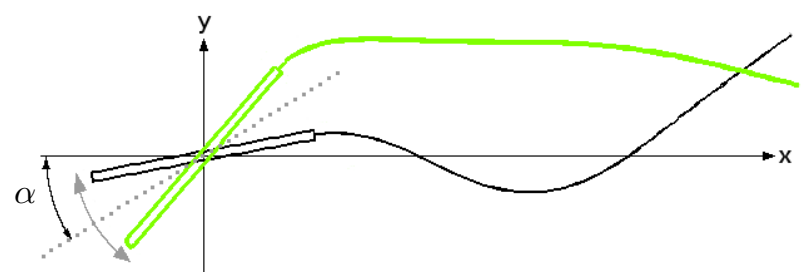

Figure 24. Superposition of front plate turning points for non-periodic oscillation

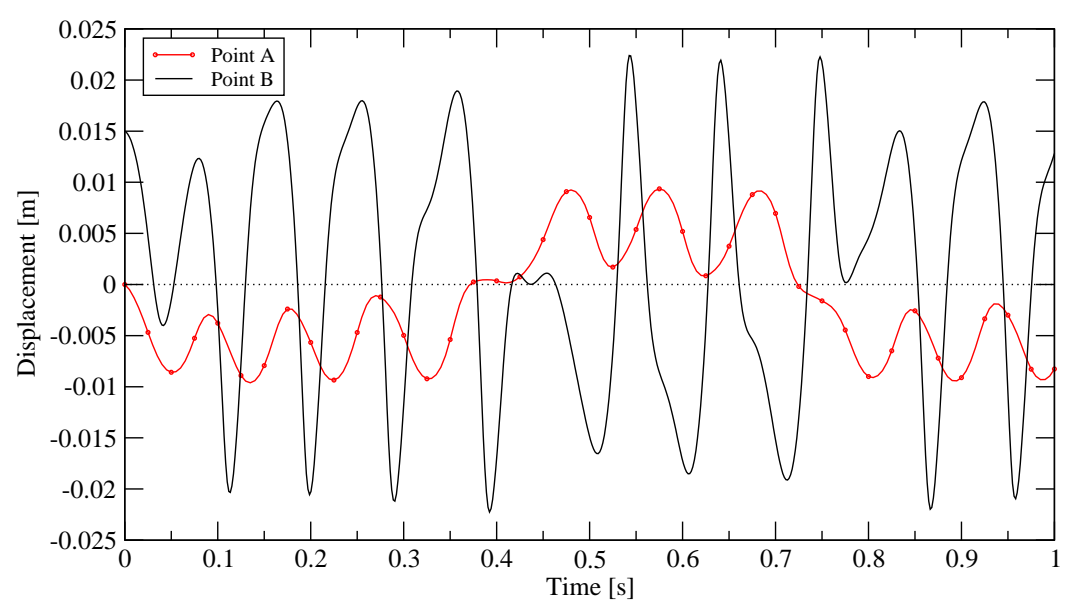

Figure 25. Non-periodic structural vibrations with very thin membrane

The non-periodic behavior disappears by increasing $D$ to $0.08 \mathrm{~mm}$ and $0.13 \mathrm{~mm}$, respectively, leading to steady small displacements of points A and B. Note that varying the membrane thickness is similar to changing its stiffness.

Next, the membrane density is varied. With a value of $\rho=\frac{1}{4} \cdot 7855 \mathrm{~kg} / \mathrm{m}^{3}(E=2$. $10^{11} \mathrm{~N} / \mathrm{m}^{2}, D=0.08 \mathrm{~mm}$ ) also steady displacements result. The same is valid for $\rho=7855 \mathrm{~kg} / \mathrm{m}^{3}$ (as mentioned before) and $\rho=4.7855 \mathrm{~kg} / \mathrm{m}^{3}$. Increasing the density 64 times to $\rho=64.7855 \mathrm{~kg} / \mathrm{m}^{3}$ a damped oscillation occurs. Finally with $\rho=$ $128.7855 \mathrm{~kg} / \mathrm{m}^{3}$ and $\rho=256.7855 \mathrm{~kg} / \mathrm{m}^{3}$ a stable periodic first swiveling motion is achieved with huge amplitudes as it can be seen in Figures 26 and 27. Figure 28 illustrates the dependency of the movement of points A and B on the membrane density. The transient regions for which the behavior changes are not clearly distinguished and are therefore indicated with dotted lines. 


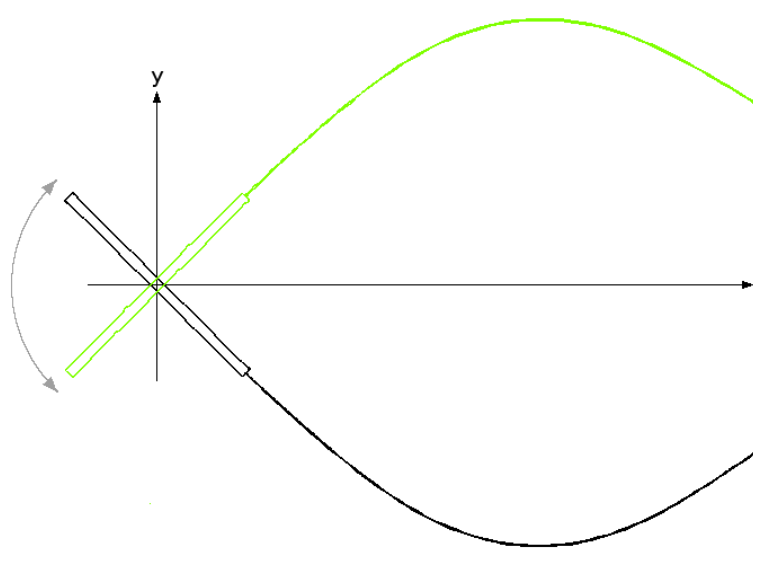

Figure 26. Superposition of front plate turning points for periodic huge oscillation

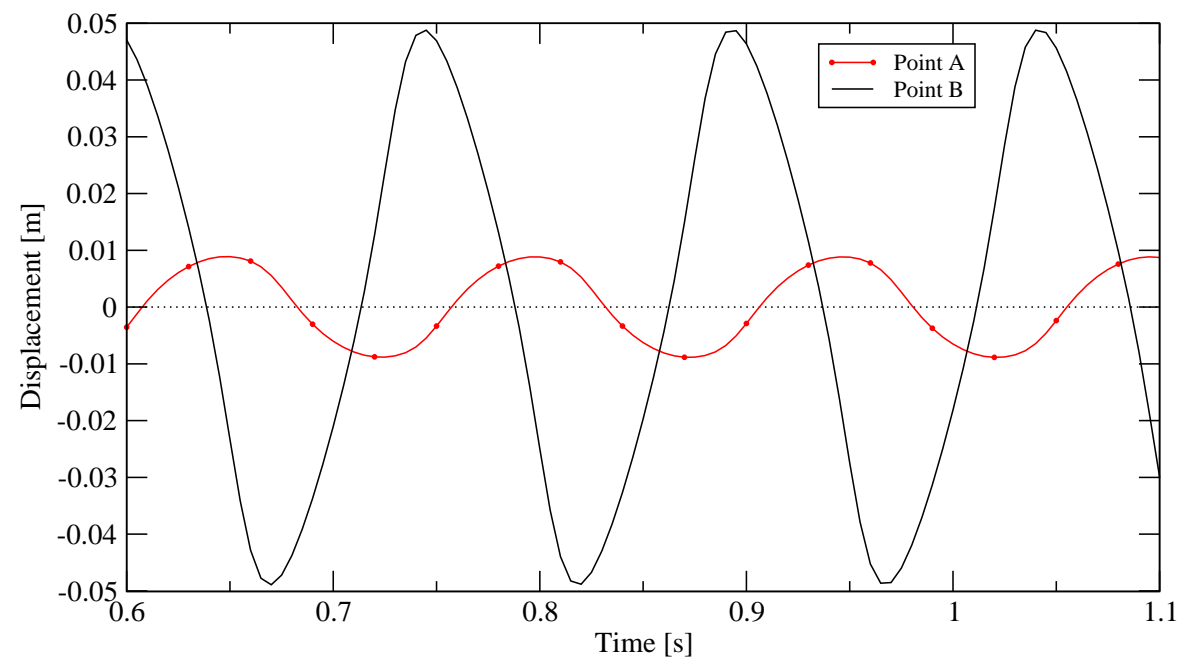

Figure 27. Periodic oscillation in first swiveling motion, configuration (a)

Figure 29 illustrates the corresponding mesh movement indicating the large deformation and the excellent grid adaption by the elliptic approach. The algebraic methods certainly will fail for this task. One can state that for configuration (a) it is only possible to get a periodic motion by increasing the density dramatically what, however, is not practicable with real materials. 
514 REMN - 16/2007. Fluid structure interaction

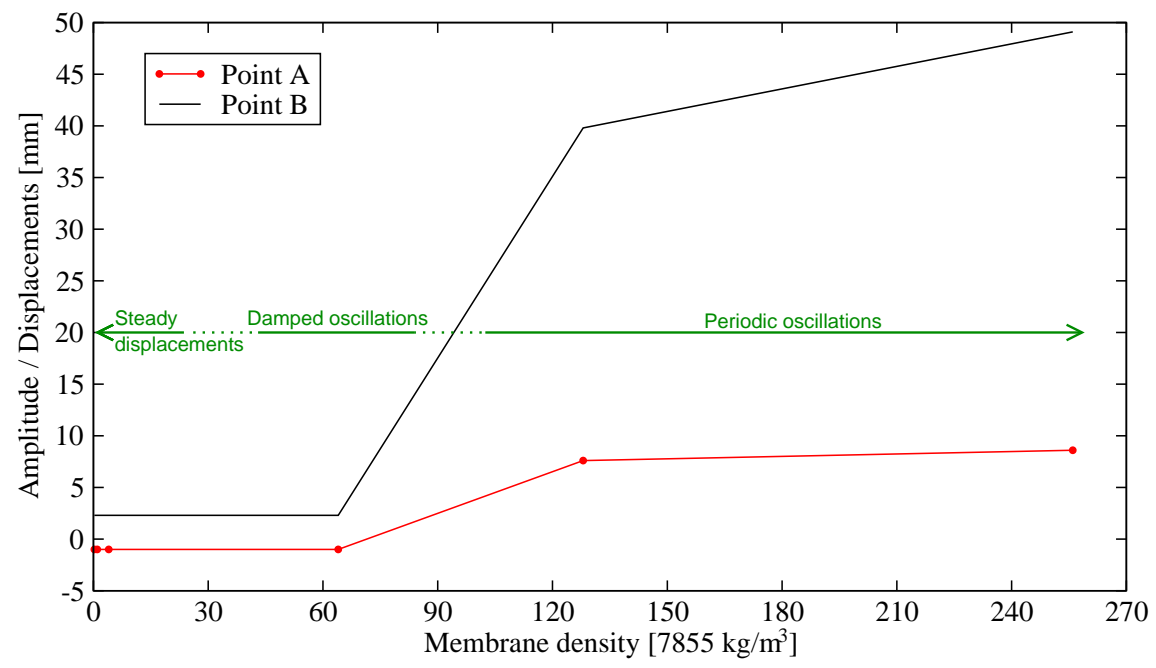

Figure 28. Dependency of the amplitudes/displacements on the membrane density

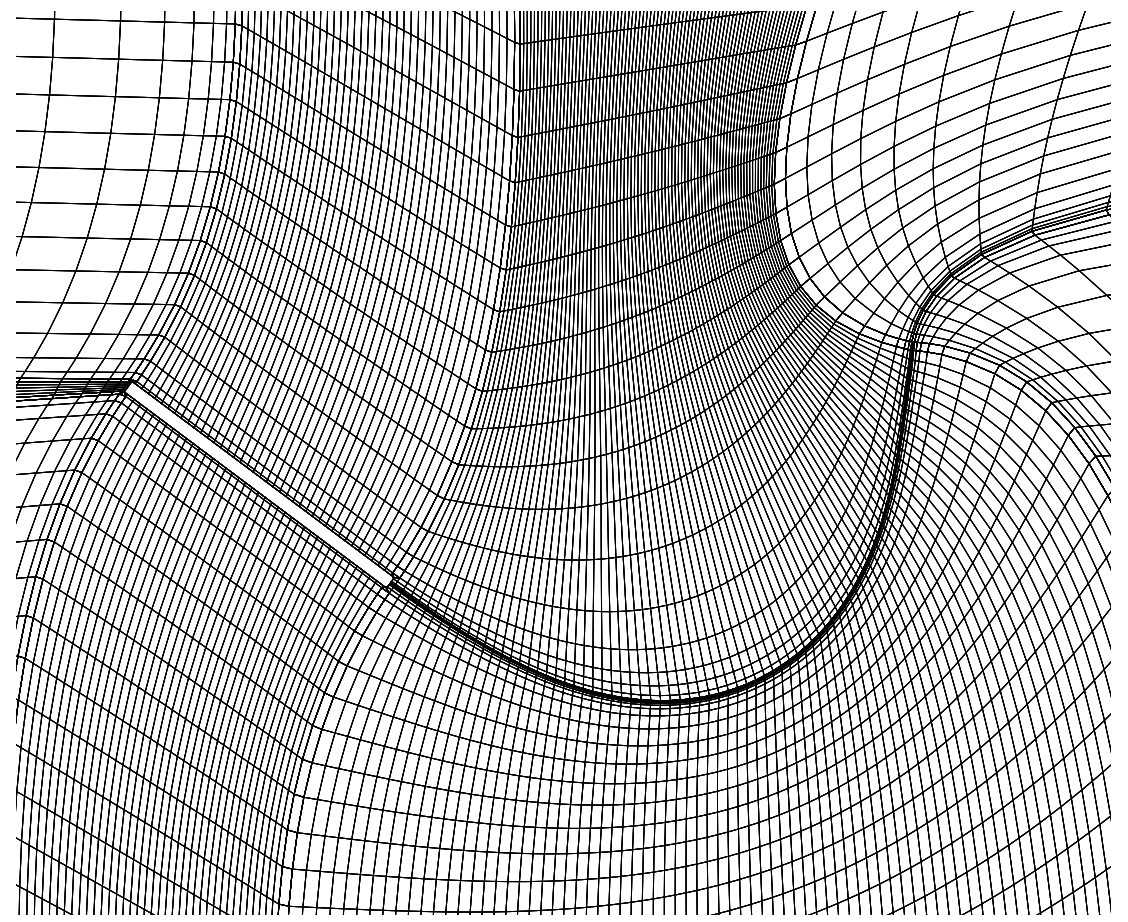

Figure 29. Mesh movement corresponding to huge structural deformation 
Next, configuration (b) is considered including a rear mass of varying density at the trailing edge. With a small mass, e.g., polyethylene with $\rho_{\mathrm{s}}=950 \mathrm{~kg} / \mathrm{m}^{3}$, all displacements are zero. Changing the mass material to steel, $\rho_{\mathrm{s}}=7855 \mathrm{~kg} / \mathrm{m}^{3}$, leads to a periodic first swiveling motion as shown in Figures 30 and 31.

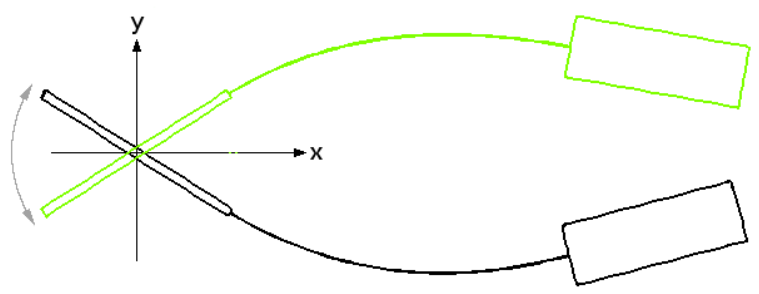

Figure 30. Superposition of images at a time-phase angle of $90^{\circ}$ and $270^{\circ}$ for periodic oscillation (first swiveling motion)

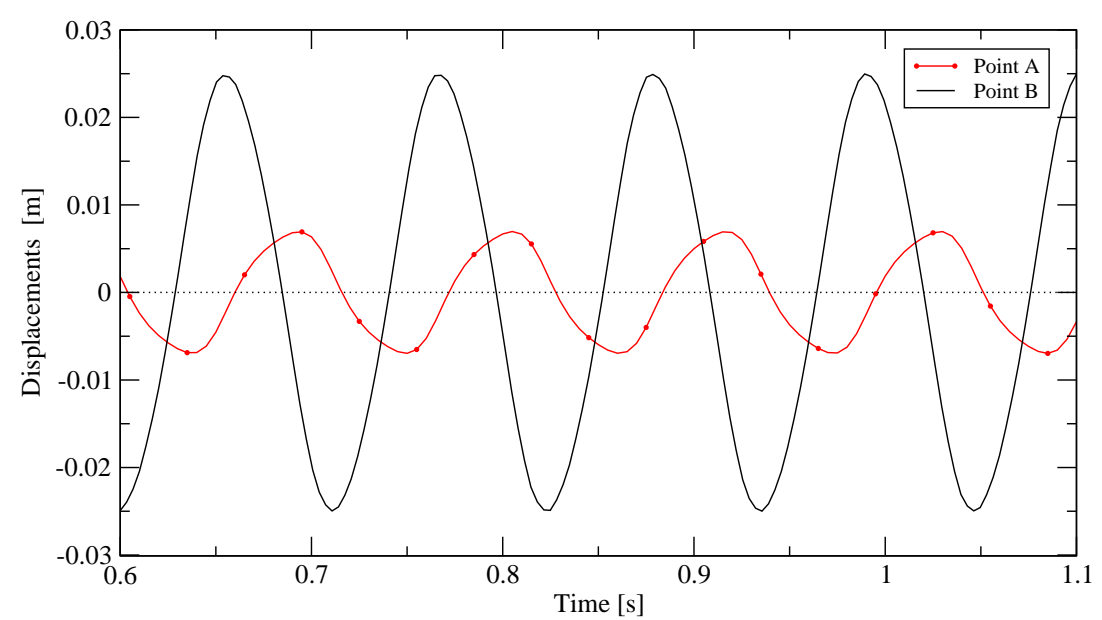

Figure 31. Periodic oscillation in first swiveling motion, configuration (b)

Finally, the membrane stiffness is varied. Decreasing the Young's modulus to $E=\frac{1}{8} \cdot 10^{11} \mathrm{~N} / \mathrm{m}^{2}$ and $E=\frac{1}{2} \cdot 10^{11} \mathrm{~N} / \mathrm{m}^{2}$, respectively, results in a periodic oscillation in the second swiveling motion as presented in Figures 32 and 33. The amplitudes decrease and the frequency rises from $8.9 \mathrm{~Hz}$ (first swiveling motion) to $13.6 \mathrm{~Hz}$ (second swiveling motion). Comparing Figures 30 and 32 with those of Section 5.1 (Figures 15,19$)$, one can recognize a similar phenomenon, especially the additional intersection point for the second swiveling motion and the increasing swiveling frequency. Applying a higher stiffness $E=8 \cdot 10^{11} \mathrm{~N} / \mathrm{m}^{2}$ leads to a damped oscillation up to zero displacements. Figure 34 illustrates how the amplitudes depend on the Young's modulus of the membrane. The transient regions are not clearly distinguished and marked 
with dotted lines therefore. Note the highly non-linear behavior, in particular the different increase and decrease of amplitudes of points A and B.

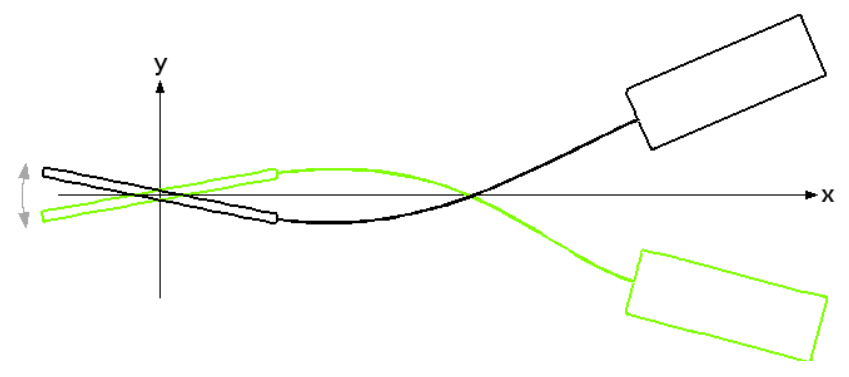

Figure 32. Superposition of images at a time-phase angle of $90^{\circ}$ and $270^{\circ}$ for periodic oscillation (second swiveling motion)

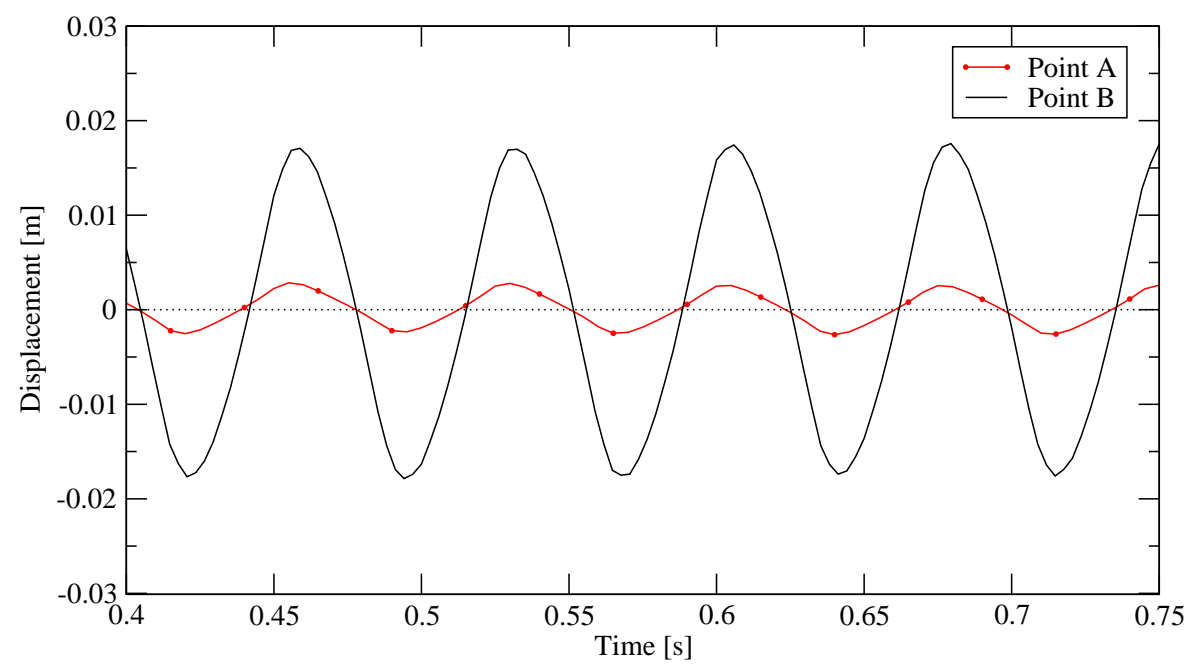

Figure 33. Periodic oscillation in second swiveling motion, configuration (b)

The results can be summarized as follows. A stable periodic swiveling motion applying real materials can only be achieved with a rear mass. Without a weight the membrane density has to be increased dramatically to achieve a stable motion. If the stiffness is too high the oscillations are damped and the displacements tend to zero. Thus, the mechanism of this fluid structure interaction configuration is that the kinematic energy of the flow is transferred to the structure which transcribes it in terms of motion (Figure 35 (1)). If the stiffness is appropriate the membrane begins to bend and the fluid damping effect is low (2a), otherwise the movement is rapidly abandoned (2b). If the density is too small the kinetic energy, stored in the structure is insufficient 
to move the membrane to a position corresponding to that where it is started at the opposite side (3a). Only if this is achieved a periodic swiveling motion is obtained (3b).

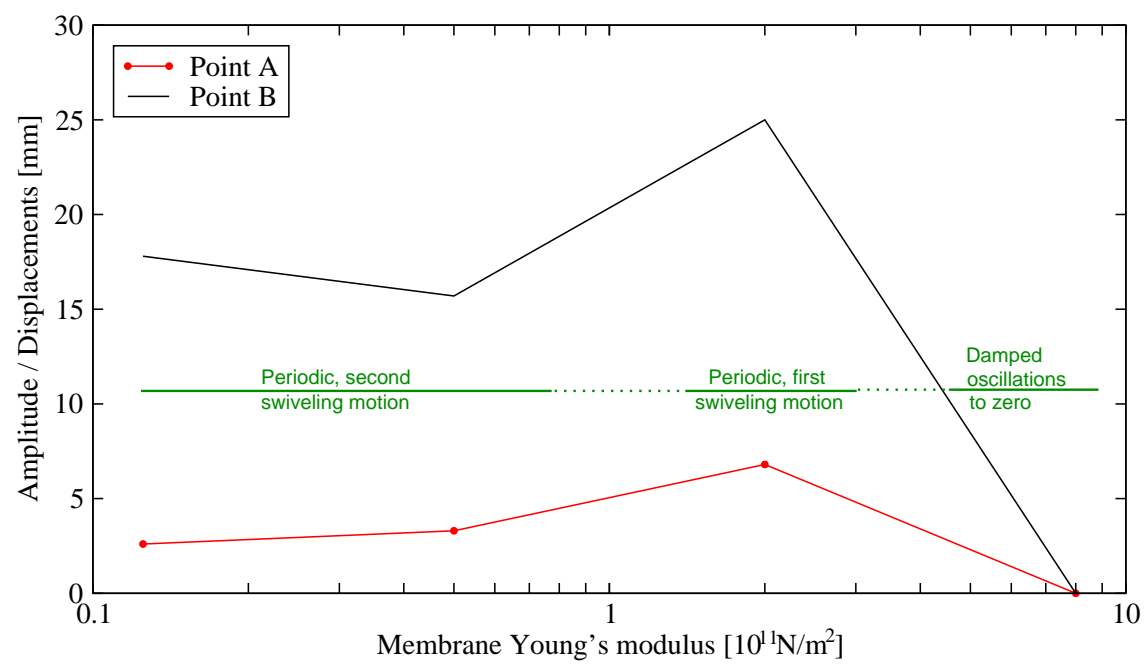

Figure 34. Dependency of the amplitude/displacement on the membrane Young's modulus, configuration (b)

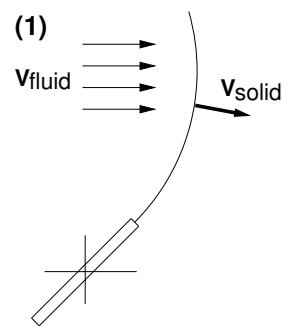

(2a)

(2b)

$\begin{array}{ll}\text { low stiffness } \rightarrow \text { low damping } & \text { high stiffness } \rightarrow \text { high damping } \\ \text { low thickness } \rightarrow \text { low damping } & \text { high thickness } \rightarrow \text { high damping }\end{array}$

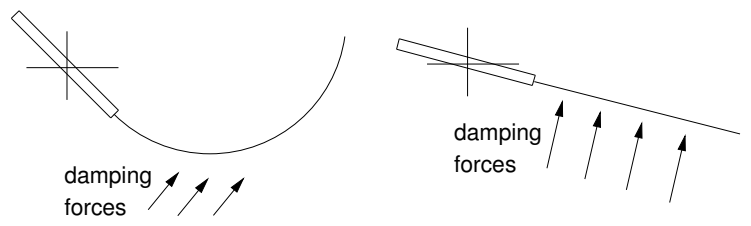

(3a)

(3b)
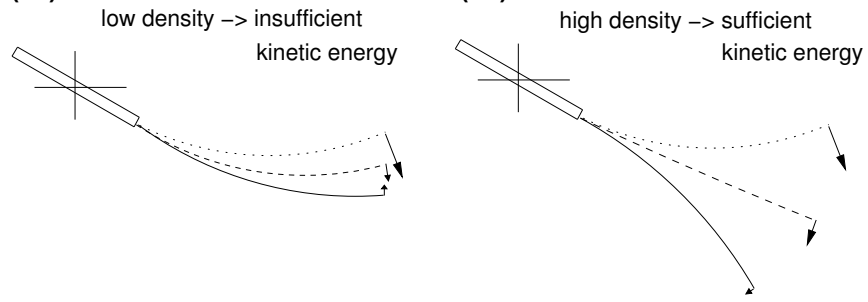

Figure 35. Mechanism of the fluid structure interaction configuration 
Table 1. Material parameters and the corresponding structural behavior

\begin{tabular}{|c|c|c|c|c|c|}
\hline \multirow{2}{*}{$\begin{array}{l}\text { Config. } \\
\text { (a) / (b) }\end{array}$} & \multicolumn{3}{|c|}{ Membrane } & \multirow{2}{*}{$\begin{array}{l}\text { Rear mass } \\
\left.\rho\left[\mathrm{kg} / \mathrm{m}^{3}\right]\right]\end{array}$} & \multirow[b]{2}{*}{ Structural behavior } \\
\hline & $\rho\left[\mathrm{kg} / \mathrm{m}^{3}\right]$ & $\mathrm{E}\left[\mathrm{N} / \mathrm{m}^{2}\right]$ & $\mathrm{D}[\mathrm{mm}]$ & & \\
\hline $\mathrm{a}$ & 7855 & $2 \cdot 10^{11}$ & 0.03 & - & non-periodic \\
\hline a & $"$ & $"$ & 0.08 & - & steady displacements \\
\hline a & $"$ & $"$ & 0.13 & - & steady displacements \\
\hline $\mathrm{a}$ & $\frac{1}{4} \cdot 7855$ & $2 \cdot 10^{11}$ & 0.08 & - & steady displacements \\
\hline $\mathrm{a}$ & 1.7855 & $"$ & $"$ & - & steady displacements \\
\hline $\mathrm{a}$ & $4 \cdot 7855$ & $"$ & $"$ & - & steady displacements \\
\hline $\mathrm{a}$ & $64 \cdot 7855$ & $"$ & $"$ & - & damped oscillation to steady displ. \\
\hline $\mathrm{a}$ & $128 \cdot 7855$ & $"$ & $"$ & - & periodic, first mode, tall amplitude \\
\hline $\mathrm{a}$ & $256 \cdot 7855$ & $"$ & $"$ & - & periodic, first mode, huge amplitude \\
\hline $\mathrm{b}$ & 7855 & $2 \cdot 10^{11}$ & 0.08 & $950(\mathrm{PE})$ & zero displacements \\
\hline $\mathrm{b}$ & $"$ & $"$ & $"$ & 7855 & periodic, first mode, moderate ampl. \\
\hline $\mathrm{b}$ & 7855 & $\frac{1}{8} \cdot 10^{11}$ & 0.08 & 7855 & periodic, second mode, moderate ampl. \\
\hline $\mathrm{b}$ & $"$ & $\frac{1}{2} \cdot 10^{11}$ & $"$ & $"$ & periodic, second mode, moderate ampl. \\
\hline $\mathrm{b}$ & $"$ & $2 \cdot 10^{11}$ & $"$ & $"$ & periodic, first mode, moderate ampl. \\
\hline $\mathrm{b}$ & $"$ & $8 \cdot 10^{11}$ & $"$ & $"$ & damped oscillation to zero \\
\hline
\end{tabular}

\section{Conclusion}

Investigations of mechanisms for fluid structure interaction configurations have been presented. Preliminary studies with respect to the grid movement illustrated the advantages of an elliptic approach, in particular in case of large mesh deformations. The complex structural behavior during fluid excited oscillations on the basis of an experimental configuration has been presented, especially the transition from a first swiveling motion to a second swiveling motion by increasing the fluid velocity. An identical phenomenon has been confirmed by other corresponding computations with excellent coincidence. In parameter studies, a variety of structural behavior, ranging from non-periodic vibrations, damped oscillations to periodic first and second swiveling motions have been found. Studying such kind of effects also gave a possibility to validate the employed implicit partitioned solution approach.

\section{References}

Demirdžić I., Perić M., “ Finite volume method for prediction of fluid flow in arbitrary shaped domains with moving boundaries", Int. J. Num. Meth. in Fluids, 10, 1990, p. 771-790.

Demirdžić I., Perić M., " Space conservation law in finite volume calculations of fluid flow", Int. J. Num. Meth. in Fluids, 8, 1998, p. 1037-1050.

FASTEST, "User Manual“, 2004. 
Gomes J. P., Lienhart H., Experimental study in a fluid-structure interaction test case, H.-J. Bungartz and M. Schäfer, LNCSE series, Springer, 2006.

Ogden R. W., Non-Linear Elastic Deformations, Dover Publications, 1997.

SCAI, "MpCCI - Mesh-Based Parallel Code Coupling Interface. User Guide V2.0“, 2004.

Schäfer M., Meynen S., Sieber R., Teschauer I., Efficiency of Multigrid Methods for the Numerical Simulation of Coupled Fluid-Solid Problems, P. Minev and Y. Lin (eds.), Scientific Computing and Applications, Advances in Computation: Theory and Practice. Nova Science Publishers, Huntington, 2001.

Spekreijse S. P., " Elliptic Grid Generation Based on Laplace Equations and Algebraic Transformations", Journal of Computational Physics, 1995, p. 38-61.

Taylor R. L., "FEAP - A Finite Element Analysis Programm. Version 7.4 User Manual“, 2002.

Taylor R. L., "FEAP - A Finite Element Analysis Programm. Version 7.5 Theory Manual", 2003.

Thompson J. F., Soni B. K., Weatherill N. P., Handbook of Grid Generation, CRC Press LLC, 1999. 
